\title{
Monitoring Quantum Simulators via Quantum Nondemolition Couplings to Atomic Clock Qubits
}

\author{
Denis V. Vasilyev $\odot,{ }^{1,2,{ }^{*}}$ Andrey Grankin,,${ }^{1,2}$ Mikhail A. Baranov, ${ }^{1,2}$ Lukas M. Sieberer, ${ }^{1,2}$ and \\ Peter Zoller (1) ${ }^{1,2}$ \\ ${ }^{1}$ Center for Quantum Physics, University of Innsbruck, 6020 Innsbruck, Austria \\ 2 Institute for Quantum Optics and Quantum Information of the Austrian Academy of Sciences, 6020 Innsbruck, \\ Austria
}

(Received 30 June 2020; revised 26 August 2020; accepted 9 September 2020; published 9 October 2020)

\begin{abstract}
We discuss monitoring the time evolution of an analog quantum simulator via a quantum nondemolition (QND) coupling to an auxiliary "clock" qubit. The QND variable of interest is the "energy" of the quantum many-body system, represented by the Hamiltonian of the quantum simulator. We describe a physical implementation of the underlying QND Hamiltonian for Rydberg atoms trapped in tweezer arrays using laser-dressing schemes for a broad class of spin models. As an application, we discuss a quantum protocol for measuring the spectral form factor of quantum many-body systems, where the aim is to identify signatures of ergodic versus nonergodic dynamics, which we illustrate for disordered one-dimensional Heisenberg and Floquet spin models on Rydberg platforms. Our results also provide the physical ingredients for running quantum phase estimation protocols for measurement of energies and preparation of energy eigenstates for a specified spectral resolution on an analog quantum simulator.
\end{abstract}

DOI: 10.1103/PRXQuantum.1.020302

\section{INTRODUCTION}

In the standard scenario of analog quantum simulation, a broad and tunable class of many-body Hamiltonians of interest is designed based on the resources provided by a particular physical platform. Examples in different physical platforms include spin models realized with Rydberg atoms [1-19] (for a review, see Ref. [20]), trapped ions [21-23] or superconducting qubits [24,25], or Hubbard models realized with bosonic and fermionic atoms in optical lattices [26-28]. The physical realization of these Hamiltonians then allows for the study of equilibrium and nonequilibrium phenomena, where the quantities of interest characterizing the quantum many-body state are spinor site-occupation correlation functions. These are inferred from (destructive) site-resolved readout of spins, or in the case of Hubbard models from quantum gas microscopy.

In contrast, we will be interested in a setting in which we learn the state and dynamics of the many-body system by entangling the state of the quantum simulator with an auxiliary quantum system, followed by measurement of the

*d.vutshi@gmail.com

Published by the American Physical Society under the terms of the Creative Commons Attribution 4.0 International license. Further distribution of this work must maintain attribution to the author(s) and the published article's title, journal citation, and DOI. auxiliary degrees of freedom, as illustrated in Fig. 1(a). In its simplest form, this auxiliary quantum system is a single qubit acting as a "clock" (as in the clock protocol [29-31]) or "control" qubit, which can be manipulated by single-qubit operations (rotations) and which we denote by "c qubit." However, the following considerations also generalize immediately to a multiqubit setting, where the auxiliary system represents a small-scale quantum memory or quantum computer.

At the heart of our considerations is the quantum nondemolition (QND) Hamiltonian

$$
\mathcal{H}_{\mathrm{QND}}=H_{\text {spin }} \otimes|0\rangle_{c}\langle 0|,
$$

which generates the QND gate

$$
\mathcal{U}_{\mathrm{QND}}(t)=\exp \left[-i \mathcal{H}_{\mathrm{QND}} t\right],
$$

entangling the quantum simulator with a $c$ qubit. To be specific, we assume a spin model with Hamiltonian $H_{\text {spin }}$ for the simulator and we denote by $\left\{|0\rangle_{c},|1\rangle_{c}\right\}$ the logical states of the $c$ qubit [32]. The Hamiltonian described previously is QND, where $H_{\text {spin }}$ is the "energy" of the quantum many-body system, which plays the role of the QND variable. To illustrate the action of the QND gate $\mathcal{U}_{\mathrm{QND}}(t)$ described previously, consider a quantum simulator prepared in superposition $\left|\psi_{\text {spin }}\right\rangle=\sum_{\ell} c_{\ell}|\ell\rangle$ of energy eigenstates, $H_{\text {spin }}|\ell\rangle=E_{\ell}|\ell\rangle$ with eigenenergies $E_{\ell}$. Under the QND gate, an initial state $|\Psi(t=0)\rangle=$ 
(a)

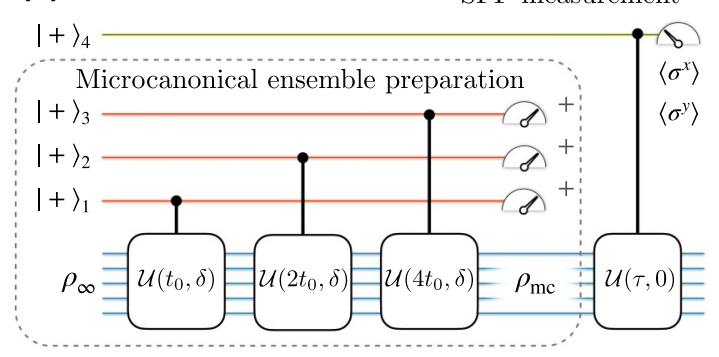

(b) Control qubit 'off'

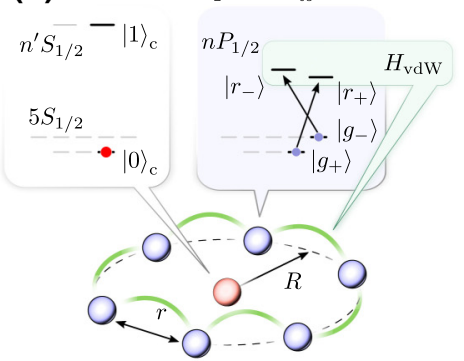

Control qubit 'on'

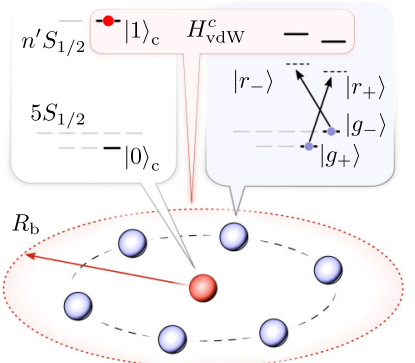

FIG. 1. (a) A quantum circuit employing a QND coupling of the quantum simulator to $c$ qubits to measure the SFF. The dashed block shows that the simulator spins (blue) initialized in the infinite-temperature ensemble $\rho_{\infty}$ are entangled with $M=3 c$ qubits (red) prepared in states $|+\rangle_{c}=\left(|0\rangle_{c}+|1\rangle_{c}\right) / \sqrt{2}$ via the QND gate $\mathcal{U}\left(t_{m}, \delta\right)=\exp \left\{-i\left[\left(H_{\text {spin }}-\delta\right) \otimes|0\rangle_{c}\langle 0|\right] t_{m}\right\}$. Postselection of the measurement results of the $c$ qubits allows us to project the spins into a microcanonical state $\rho_{\mathrm{mc}}$. The simulator in the state $\rho_{\mathrm{mc}}$ is once again entangled with the $c$ qubit (green) to measure the SFF (see Sec. III). Alternatively, the protocol can be realized with a single $c$ qubit via sequential entanglement and measurement cycles. (b) Engineering the QND Hamiltonian (1) with Rydberg-dressed atoms. The left panel shows that the $c$ qubit (red) in the state $|0\rangle_{c}$ does not affect the evolution of the simulator spins (blue) arranged in a ring of radius $R$ around the control atom. The spins (separated by a distance $r$ ) are represented by the hyperfine ground states $\left|g_{ \pm}\right\rangle$of ${ }^{87} \mathrm{Rb}$ and interact via virtually excited Rydberg states $\left|r_{ \pm}\right\rangle$. The right panel shows that the $c$ qubit excited to the Rydberg state $|1\rangle_{c}$ breaks the dressing scheme for the simulator atoms within the Rydberg-blockade radius $R_{b}$, thus blocking the free evolution and realizing the controlled unitary $\mathcal{U}_{\mathrm{QND}}(t)$.

$\left|\psi_{\text {spin }}\right\rangle \otimes|+\rangle_{c}$ of the joint quantum simulator prepared in $\left|\psi_{\text {spin }}\right\rangle$ and the $c$ qubit prepared in the superposition state $|+\rangle_{c}=\frac{1}{\sqrt{2}}\left(|0\rangle_{c}+|1\rangle_{c}\right)$ will evolve according to

$$
\begin{aligned}
|\Psi(t=0)\rangle \rightarrow & |\Psi(t)\rangle=\mathcal{U}_{\mathrm{QND}}(t)|\Psi(0)\rangle \\
& =\sum_{\ell} c_{\ell}|\ell\rangle \otimes \frac{1}{\sqrt{2}}\left(e^{-i E_{\ell} t}|0\rangle_{c}+|1\rangle_{c}\right) .
\end{aligned}
$$

Thus the superposition of many-body energy eigenstates gets entangled with the phase of the Bloch vector of the $c$ qubit rotating in the $x-y$ plane on the Bloch sphere. A readout of this phase via the $c$ qubit provides us with a QND measurement of the "energy" of the quantum many-body system. In a broader context, we note that this QND gate is also the basic building block of quantum algorithms such as quantum phase estimation (QPE) [33-35] to measure the energies of the many-body spin system and prepare corresponding eigenstates with a prescribed spectral resolution. We use such features in a protocol to measure the spectral form factor (SFF), where we access correlations between eigenenergies $E_{\ell}$, encoded through a Fourier transform, via the $c$ qubit.

In this paper, we will first address the challenge of implementing the QND Hamiltonian (1) and QND gate for a broad class of freely designable spin models in a quantum simulator in atomic physics setups. Remarkably, as we show in Sec. II, this can be achieved with Rydberg-tweezer platforms [1-5,8,9] using laser-dressing schemes [12-17] and by employing a Rydberg-blockade mechanism between the $c$ qubit and the simulator atoms to implement the QND Hamiltonian (1). Second, we wish to explore and illustrate the application of quantum protocols that build on the QND gate described previously, providing access to quantum many-body observables of interest under experimentally realistic conditions. A relevant example is provided by measurement of the SFF, as discussed in the context of Refs. [36-39], where the aim is to identify signatures of ergodic versus nonergodic dynamics without an explicit spectroscopic study [40-42] of the energy spectrum. In Sec. III, we describe a protocol in which the SFF can be measured via QND couplings to a $c$ qubit. We illustrate this protocol and its performance with simulated measurement runs for the disordered one-dimensional (1D) Heisenberg model and for Floquet models, which can be implemented using our techniques on Rydberg platforms.

The paper is organized as follows. In Sec. II, we discuss implementation of the QND Hamiltonian with Rydbergtweezer arrays. The properties of the SFF in Hamiltonian systems are briefly summarized in Sec. III, where we also present the protocol for preparation of the initial state (Sec. III B1) and the protocol for the SFF measurement, together with discussion of its experimental limitations (Sec. III B2). The SFF of Floquet systems and the corresponding measurement protocol are considered in Sec. IV and we conclude in Sec. V.

\section{PHYSICAL REALIZATION OF $\mathcal{H}_{\text {QND }}$ IN RYDBERG-TWEEZER ARRAYS}

The challenge is to implement $\mathcal{H}_{\mathrm{QND}}$ [Eq. (1)] for a broad and tunable class of spin models $H_{\text {spin }}$ on a given physical platform. The following relevant example is the 
disordered 1D Heisenberg spin-1/2 model,

$$
H_{\text {spin }}=\sum_{i<j=1}^{L} \sum_{\eta=x, y, z} J_{i j}^{(\eta)} \sigma_{i}^{\eta} \sigma_{j}^{\eta}+\sum_{i=1}^{L} h_{i}^{z} \sigma_{i}^{z},
$$

or, focusing on a specific choice of the couplings used in the following, we have

$$
\begin{aligned}
H_{\text {spin }}= & J \sum_{i=1}^{L}\left\{\left(\sigma_{i}^{x} \sigma_{i+1}^{x}+\sigma_{i}^{y} \sigma_{i+1}^{y}+\Delta \sigma_{i}^{z} \sigma_{i+1}^{z}\right)\right. \\
& \left.+J_{2}\left(\sigma_{i}^{x} \sigma_{i+2}^{x}+\sigma_{i}^{y} \sigma_{i+2}^{y}\right)+\Delta_{2} \sigma_{i}^{z} \sigma_{i+2}^{z}\right\} \\
& +\sum_{i=1}^{L} h_{i}^{z} \sigma_{i}^{z}
\end{aligned}
$$

with designable single-particle and two-body (interaction) terms. In implementing $\mathcal{H}_{\mathrm{QND}}$ we are required to implement the two- and three-body terms involving the $c$ qubit as $\mathcal{H}_{\mathrm{QND}}=H_{\text {spin }} \otimes|0\rangle_{c}\langle 0|$.

Rydberg-tweezer arrays in one, two, and three dimensions $[1-5,8,9]$ provide the tools to design a broad class of spin Hamiltonians $H_{\text {spin }}$ via Rydberg dressing [1217]. Here, long-lived atomic (hyperfine) ground states are trapped in the optical tweezer and play the role of the spins in our quantum simulator, while the two-body interactions are engineered by admixing weakly to the ground state via off-resonant laser dressing of the (strong) van der Waals interactions between Rydberg states [43,44]. Remarkably, the same "dressing toolbox" that allows us to design $H_{\text {spin }}$ also provides us with a recipe to engineer $\mathcal{H}_{\mathrm{QND}}$.

Engineering of two-qubit entangling gates using a Rydberg blockade is a well-established field [18,45-49]. Here, we are interested in a multiqubit QND gate where (as originally discussed in Ref. [50]; see also Refs. [51-56]) interactions engineered via laser dressing can be turned on and off by preparing a $c$ qubit in the ground state $|0\rangle_{c}$ or a Rydberg state $|1\rangle_{c}$, respectively [see Fig. 1(b), left and right panels, respectively]. If the $c$ qubit is in the ground state, it does not interact with the system spins and thus the Hamiltonian $H_{\text {spin }}$ is realized, as discussed previously. On the other hand, for the $c$ qubit in the Rydberg state $|1\rangle_{c}$, the long-range character and strength of Rydberg-Rydberg interactions will, via the dipole blockade mechanism, detune the Rydberg states of the simulator atoms, thus effectively turning off the dressing interactions, i.e., we have $\mathcal{H}_{\mathrm{QND}}=H_{\text {spin }} \otimes|0\rangle_{c}\langle 0|$. We analyze this in the following in a realistic atomic physics setting.

\section{A. Hamiltonian for the simulator and $c$ qubit}

We consider the setup outlined in Fig. 1(b). $L$ atoms trapped in optical tweezers (with trapping frequency $\omega_{\text {trap }}$ ) are arranged in a ring representing a $1 \mathrm{D}$ quantum simulator of spin $1 / 2$ with periodic boundary conditions. The distance $r$ between simulator atoms is assumed to be larger than $2.4 \mu \mathrm{m}$ as discussed in Appendix A3. The simulator atoms are assumed to be close to their motional ground state. We also consider the effective spin dynamics to be adiabatic with respect to the atomic motion, $J_{i j} \ll \omega_{\text {trap }}$, and thus neglect the latter. This condition is easily satisfied in the considered setup.

The $c$ qubit is represented by an atom trapped in the center of the ring (more $c$ qubits can be realized with atoms placed, for example, on the axis of the ring). In Fig. 1(b), we show the atomic level structure for the atoms representing the quantum simulator and the control atom. The spin$1 / 2$ degrees of freedom of the simulator are encoded in two long-lived hyperfine ground states $\left|g_{ \pm}\right\rangle$with energies $E_{g \pm}$. These are coupled to Rydberg states $\left|g_{ \pm}\right\rangle \rightarrow\left|r_{ \pm}\right\rangle$with energies $E_{r \pm}$ by two off-resonant lasers with respective frequencies $\omega_{ \pm}$and detunings $\Delta_{ \pm}=\omega_{ \pm}-E_{r \pm}+E_{g \pm} \gg$ $\Omega_{ \pm}$, and corresponding Rabi frequencies $\Omega_{ \pm}$. The ground state of the $c$ qubit is $|0\rangle_{c}$ with energy $E_{g c}$, while $|1\rangle_{c}$ is a Rydberg state with energy $E_{r c}$. We drive the control-atom transition with a laser of frequency $\omega_{c}$, which is tuned near resonance with detuning $\Delta_{c}=\omega_{c}-E_{c r}+E_{g c}$. The corresponding Rabi frequency is $\Omega_{c}$. In our protocol, the $c$ qubit is prepared initially in a superposition state of a ground and an excited state with a $\pi / 2$ pulse.

The Hamiltonian of the total system is $H_{\text {tot }}=H_{s}+$ $H_{c}+H_{s c}$, written as the sum of the simulator- and controlatom Hamiltonians, and the simulator-control interaction. These are given by

$$
\begin{aligned}
H_{s}= & \left\{\sum _ { i = 1 } ^ { L } \sum _ { \alpha = \pm } \left[E_{g_{\alpha}}\left|g_{\alpha}\right\rangle_{i}\left\langle g_{\alpha}\left|+E_{r_{\alpha}}\right| r_{\alpha}\right\rangle_{i}\left\langle r_{\alpha}\right|\right.\right. \\
& \left.\left.+\left(\Omega_{\alpha}\left|g_{\alpha}\right\rangle_{i}\left\langle r_{\alpha}\right| e^{-i \omega_{\alpha} t}+\text { H.c. }\right)\right]+\sum_{i<j}^{L} H_{\mathrm{vdW}}^{(i, j)}\right\} \otimes \mathbb{I}_{c} \\
H_{c}= & \mathbb{I}_{s} \otimes\left[E_{g c}|0\rangle_{c}\left\langle 0\left|+E_{c r}\right| 1\right\rangle_{c}\langle 1|\right. \\
& \left.+\left(\Omega_{c}|0\rangle_{c}\langle 1| e^{-i \omega_{c} t}+\text { H.c. }\right)\right], \\
H_{s c}= & \sum_{i=1}^{L} H_{\mathrm{vdW}}^{(i, c)} .
\end{aligned}
$$

Here, $H_{\mathrm{vdW}}^{(i, j)}$ is the van der Waals interaction between the Rydberg manifolds $\left\{\left|r_{ \pm}\right\rangle_{i}\right\}$ and $\left\{\left|r_{ \pm}\right\rangle_{j}\right\}$ of simulator atoms $i$ and $j$, and $H_{\mathrm{vdW}}^{(i, c)}$ denotes the van der Waals interaction between the control atom in $|1\rangle_{c}$ and Rydberg atom $\left\{\left|r_{ \pm}\right\rangle_{i}\right\}$.

As stated previously, in our model, the effective spin $1 / 2$ of the quantum simulator is encoded in ground states $\left|g_{ \pm}\right\rangle$of the simulator atoms ( for a specific choice, see, 
e.g., Refs. [43,57]). Following Ref. [43], an effective spinspin interaction is obtained by admixing to these ground states with a laser a fine-structure split Rydberg state. We note that it is the combination of a fine-structure resolved Rydberg manifold (i.e., the spin-orbit coupling) together with the van der Waals interaction (away from Förster resonances [48]) that provides the physical mechanism for the effective spin-spin interactions in the groundstate manifold and determines the spin models that can be realized in this setup (see Appendices A and B). To be specific, we assume below ${ }^{87} \mathrm{Rb}$ with Rydberg states $\left|r_{ \pm}\right\rangle=\left|n P_{1 / 2}, m_{j}= \pm 1 / 2\right\rangle$ for the simulator atoms and the Rydberg state of the control atom $\left|n^{\prime} S_{1 / 2}, m_{j}=1 / 2\right\rangle$. We furthermore assume $\left|n-n^{\prime}\right| \gg 1$ in order to avoid direct dipolar exchange interaction between control and simulator atoms. In the basis $\left\{\left|r_{ \pm}\right\rangle_{i} \otimes\left|r_{ \pm}\right\rangle_{j}\right\}$ of the given Rydberg manifold, the interaction $H_{\mathrm{vdW}}^{(i, j)}$ is then represented by a $4 \times 4$ matrix [57],

$$
H_{\mathrm{vdW}}^{(i, j)}=\frac{1}{r_{i j}^{6}}\left[C_{6} \mathbb{I}_{4}-\widetilde{C}_{6} \mathbb{D}_{0}\left(\theta_{i j}, \phi_{i j}\right)\right] .
$$

Here, $C_{6}$ and $\widetilde{C}_{6}$ are van der Waals interaction constants, $\mathbb{I}_{4}$ is the identity matrix, and $\mathbb{D}_{0}$ a $4 \times 4$ matrix, with $\theta_{i j}$, $\phi_{i j}$ angles representing the axis connecting the pair of atoms $i, j$ with distance $r_{i j}$. Explicit expressions for these quantities are provided in Appendix A1. In a similar way, the van der Waals interaction between the simulator atom $i$ and the control atom $c$ has the form

$$
H_{\mathrm{vdW}}^{(i, c)} \approx \frac{C_{6}^{\prime}}{r_{i c}^{6}} \sum_{\alpha= \pm}\left|r_{\alpha}\right\rangle_{i}\left\langle r_{\alpha}|\otimes| 1\right\rangle_{c}\langle 1|,
$$

where $C_{6}^{\prime}$ is the corresponding van der Waals coefficient. Here, we make the assumption that $C_{6}^{\prime}$ is independent of the state $\left|r_{ \pm}\right\rangle$(see Appendix A2). Thus, conditional on the control atom being in the Rydberg state $|1\rangle_{c}$, the Rydberg energies of the simulator atoms are shifted by $E_{r \pm} \rightarrow$ $E_{r \pm}+C_{6}^{\prime} / r_{i c}^{6}$, which for the ring geometry of Fig. 1(b) provides an identical shift for all simulator atoms. In the spirit of the Rydberg-blockade mechanism, we assume this shift to be large. Thus, for a control atom in the ground state $|0\rangle_{c}$, the dressing lasers $\omega_{ \pm}$are detuned from the Rydberg states $\left|r_{ \pm}\right\rangle_{i}$ by $\Delta_{ \pm}$, while the presence of a control atom in $|1\rangle_{c}$ will detune these excited states by $\Delta_{ \pm}-C_{6}^{\prime} / r_{i c}^{6}$. For a control atom in $|0\rangle_{c}$, the dressing lasers will thus induce interactions between the effective ground-state spin $1 / 2$ by admixing the Rydberg-Rydberg interactions $H_{\mathrm{vdW}}^{(i, j)}$ to the ground-state manifold, while a control atom in $|1\rangle_{c}$ detunes the Rydberg states and thus effectively shuts off Rydberg dressing.

\section{B. QND Hamiltonian and imperfections}

The derivation of $\mathcal{H}_{\mathrm{QND}}$ now proceeds by a perturbative elimination of the Rydberg manifold of the simulator (a)

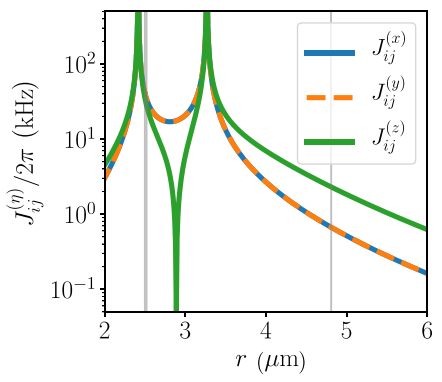

(b)

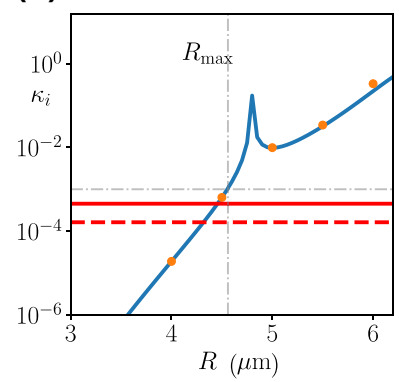

FIG. 2. The engineered QND Hamiltonian. (a) The spin-spin interaction constants as a function of the interatomic distance $r$. The gray vertical lines indicate the nearest- and next-nearestneighbor positions. (b) The decoherence parameters $\kappa_{1}$ (red solid), $\kappa_{2}$ (red dashed), and $\kappa_{3}$ (analytical, blue solid line; numerical, orange dots) for $L \sim 10$ spins. The parameters of the dressing are $\xi_{ \pm} \equiv \Omega_{ \pm} / \Delta_{ \pm}=0.2$ and $\Delta_{ \pm}=-9 \mathrm{MHz}$. The principal quantum numbers of the Rydberg atoms are $n=60$ and $n^{\prime}=71$.

atoms. First, for the control atom in the ground state $|0\rangle_{c}$ and $\Omega_{c}=0$, the dressing lasers for the simulator atoms are described by the detunings $\Delta_{ \pm}$, as discussed previously and the relevant perturbation parameter for elimination of the Rydberg states is $\xi_{ \pm}=\Omega_{ \pm} / \Delta_{ \pm} \ll 1$. This yields an effective dynamics as a spin- $1 / 2$ model for the dressed ground states $\left|\tilde{g}_{ \pm}\right\rangle_{i}$, with an effective spin-1/2 Hamiltonian denoted by $H_{\text {spin }}$. The design of the desired spin Hamiltonians via dressing schemes by an appropriate choice of the atomic and laser configurations, and the van der Waals interaction for a given geometry, have been discussed in Ref. [43]. In Appendix B, we provide a derivation for the disordered 1D Heisenberg chain in the form (3). There, we list explicit expressions for the $J^{(x, y, z)}, h$ etc. in Eqs. (B9)-(B12) of Appendix B as functions of the microscopic atomic parameters. Figure 2(a) shows the values of the spin-spin interaction constants $J_{i j}^{(\eta)}$ as a function of the interatomic distance for a particular choice of dressing-scheme parameters.

Second, we repeat this derivation for the control atom in the excited state $|1\rangle_{c}$, which amounts to replacing the detunings $\Delta_{ \pm} \rightarrow \Delta_{ \pm}-C_{6}^{\prime} / r_{i c}^{6}$ in the expressions for the parameters of the Hamiltonian (B6) of Appendix B. This results in a spin Hamiltonian that we denote by $H_{\text {spin }}^{\prime}$, with the same structure as $H_{\text {spin }}$ but strongly suppressed couplings $J^{\prime} \ll J$ etc. After combining the two cases, we obtain the Hamiltonian describing the coupling between the simulator and the $c$ qubit:

$$
\tilde{\mathcal{H}}=H_{\text {spin }} \otimes|0\rangle_{c}\left\langle 0\left|+H_{\text {spin }}^{\prime} \otimes\right| 1\right\rangle_{c}\langle 1|
$$

If $H_{\text {spin }}$ and $H_{\text {spin }}^{\prime}$ commute, $\tilde{\mathcal{H}}$ is equivalent to the QND Hamiltonian (1) with $H_{\text {spin }} \rightarrow H_{\text {spin }}-H_{\text {spin }}^{\prime}$. In the opposite case, $H_{\text {spin }}^{\prime}$ results in errors in the entangling gate 
$\mathcal{U}_{\mathrm{QND}}(t)$, with the error rate characterized by a dimensionless parameter $\kappa_{3}=\left|H_{\text {spin }}^{\prime}\right| /\left|H_{\text {spin }}\right|$, where $|\ldots|$ denotes the difference between the maximal and the minimal eigenvalues of the corresponding operator. Another source of error is related to spontaneous emission of Rydberg states of the control and simulator atoms, with error rates characterized by $\kappa_{1} \equiv \gamma_{d}^{\prime} /\left|H_{\text {spin }}\right|$ and $\kappa_{2} \equiv \xi_{ \pm}^{2} \gamma_{d} L /\left|H_{\text {spin }}\right|$, respectively, where $\gamma_{d}^{\prime}$ and $\gamma_{d}$ are the corresponding spontaneous emission rates [for more details, see Fig. 2(b) and Appendix D]. The largest error rate determines the coherence time $t_{\text {coh }} \sim\left[\left|H_{\text {spin }}\right| \max \left\{\kappa_{i}\right\}\right]^{-1}$, below which the errors in the gate operation due to spontaneous emission and $H_{\text {spin }}^{\prime}$ can be ignored.

Thus, for $t<t_{\mathrm{coh}}$, the quantum simulator coupled to the $c$ qubit can be described by the QND Hamiltonian given in Eq. (1):

$$
\tilde{\mathcal{H}}_{\mathrm{QND}}=\left(H_{\mathrm{spin}}-\delta\right) \otimes|0\rangle_{c}\langle 0|,
$$

which is written here in the rotating frame with respect to the laser of the $c$ qubit, and we set $\Omega_{c}=0$ in $H_{c}\left(\Omega_{c} \neq 0\right.$ is needed only during the preparation stage of the $c$ qubit and for the measurement readout). In the previous equation, $\delta$ refers to a renormalized detuning of the control laser (see Appendix B).

The geometric constraints on the possible configurations of atoms in space are imposed by the validity conditions of the van der Waals interaction Hamiltonians (7) and (8) and by the efficiency of the Rydberg blockade. In particular, the distances between the control and simulator atoms $R$ and between the simulator atoms $r$ are limited to $R<R_{b} \approx 6.5 \mu \mathrm{m}$ and $r \gtrsim 2.4 \mu \mathrm{m}$, respectively (see Appendices A3 and D). This restricts the number of atoms in $1 \mathrm{D}$ ring geometry to 12 . Larger system sizes can be achieved in two-dimensional (2D) or quasi-2D arrangements of atoms. For example, the number of atoms can be doubled by considering two coaxial rings of atoms. We note that in this case the resulting spin model parameters are not described by Fig. 2(a) due to a more complex van der Waals interaction pattern for inter-ring pairs of atoms. Alternatively, atoms can form a small 2D lattice fitting into the blockade radius $R_{b}$ of the control atom placed above the lattice.

\section{MEASUREMENT OF THE SFF IN HAMILTONIAN SYSTEMS}

For a generic quantum many-body system, the energylevel statistics are an indicator that allows us to distinguish between quantum ergodic and nonergodic regimes [5861]. Quantum ergodic (or chaotic) systems are characterized by eigenenergy statistics given by the Wigner-Dyson distribution [62] in a universality class of random matrices and the eigenfunctions are delocalized over the configuration space. In contrast, nonergodic quantum systems, such as integrable models, display Poisson statistics of energy levels and localized wave functions. These considerations apply not only to systems with time-independent Hamiltonians and their energy spectrum but also to time-periodic Floquet systems [58] characterized by quasienergies.

An equivalent characterization of ergodic versus nonergodic dynamics is provided by the SFF, where spectral features are displayed in the time domain, i.e., essentially as the Fourier transform of the two-point correlation function of the spectral density. For a many-body Hamiltonian $H_{\text {spin }}$ with eigenenergies $E_{\ell}$, the SFF is defined as

$$
\begin{aligned}
K(\tau) & =\overline{\left|\sum_{\ell} f\left(E_{\ell}\right) e^{-i E_{\ell} \tau}\right|^{2}} \\
& \equiv \overline{\left|\operatorname{tr}\left(e^{-i H_{\mathrm{spin}} \tau} \rho_{\mathrm{mc}}\right)\right|^{2}} .
\end{aligned}
$$

Here, $f(E)$ is a non-negative normalized smooth filter function of width $\Delta E$ covering a band in the middle of the energy spectrum, where the density of states is flat, thus probing the properties of typical states of the many-body system while eliminating contributions from spectral edges. The overline indicates a possible disorder average. The second line of (10) rewrites the SFF in terms of the microcanonical density operator $\rho_{\mathrm{mc}}=$ $\sum_{\ell} f\left(E_{\ell}\right)|\ell\rangle\langle\ell|$ representing an initial density matrix. The SFF has been central in high-energy physics [36] and in the recent discussion of the transition from many-body localization (MBL) to quantum chaos in a class of generic spin chains with disorder [37-39].

\section{A. Properties of the SFF}

To illustrate the generic behavior of SFF, we show in Fig. 3 a numerically calculated SFF for the disordered Heisenberg $X X Z$ spin-1/2 chain of length $L=12$ with Hamiltonian (3). A random local transverse field [the last term in Eq. (3)] plays the role of disorder, with values $h_{i} \in[-W, W]$ drawn from a uniform distribution and $W$ characterizing the disorder strength. We assume periodic boundary conditions and consider the sector with zero total spin projection on the $z$ axis, $S_{z}=\sum_{i=1}^{L} \sigma_{i}^{z}=0$. The choice of this model is based on the following considerations. First, it exhibits a transition from quantum chaos for weak disorder to many-body localization for strong disorder and, second, it belongs to the class of models in which the QND gate and thus the SFF protocol of the following section can be implemented with the dressing scheme in Rydberg-tweezer arrays (see Sec. II).

According to Fig. 3, for small times $\tau$ the SFF $K(\tau)$ decays from its initial value $K(0)=1$ due to dephasing on a time scale of approximately $1 / \Delta E$. Here, $\Delta E \approx\left(E_{\max }-\right.$ $\left.E_{\min }\right) / 6$, i.e., $1 / 6$ of the width of the energy spectrum. The signature of quantum chaos is the existence of a ramp at long times $\tau$ : random-matrix theory (RMT) for the Gaussian orthogonal ensemble (GOE), which is applicable to 


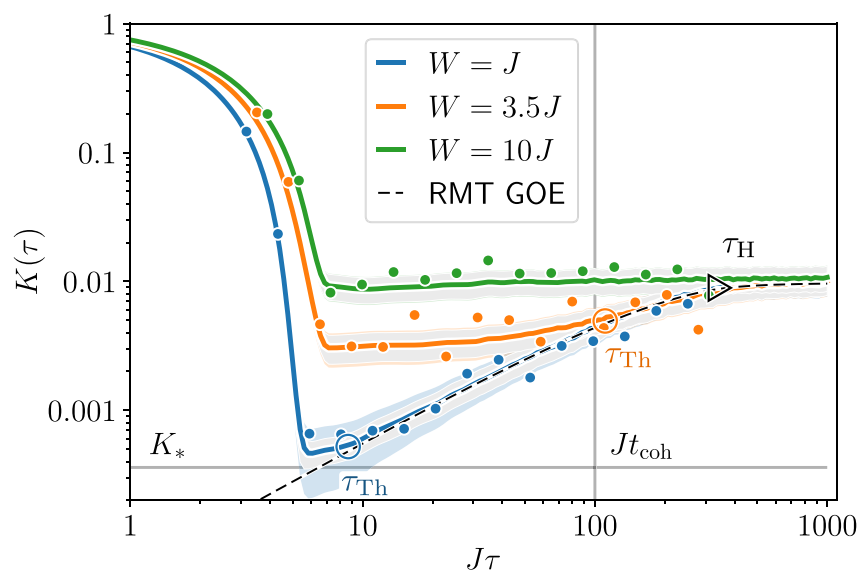

FIG. 3. The measurement of the spectral form factor $K(\tau)$ in the Heisenberg chain (3) of $L=12$ spins for different disorder strengths $W$. The black dashed line represents the RMT prediction $K_{\mathrm{GOE}}(\tau)(12)$. The colored dots show the results of the simulated measurements of $K(\tau)$. The colored solid lines and the shaded areas indicate, respectively, the numerical prediction for $K(\tau)$ and the root-mean-square error due to the shot noise and disorder averaging for the simulated $\simeq 2 \times 10^{5}$ experimental runs per data point. The gray areas show the contribution of averaging over 20 disorder realizations. The colored circles indicate the Thouless times $\tau_{\mathrm{Th}}$ and the black triangle shows the Heisenberg time $\tau_{H}$. The parameters of the Heisenberg model (3) are $\Delta=0.8, J_{2}=0.02$ and $\Delta_{2}=0.06$.

systems that obey time-reversal symmetry, predicts

$K_{\mathrm{GOE}}(\tau)=K_{\infty} \begin{cases}2 \frac{\tau}{\tau_{H}}-\frac{\tau}{\tau_{H}} \log \left(1+2 \frac{\tau}{\tau_{H}}\right), & 0<\tau \leq \tau_{H}, \\ 2-\frac{\tau}{\tau_{H}} \log \left(\frac{2 \tau+\tau_{H}}{2 \tau-\tau_{H}}\right), & \tau>\tau_{H},\end{cases}$

which is shown as a dashed line in Fig. 3. In Eq. (12), $\tau_{H}=2 \pi / \delta_{E} \sim 2^{L} / L$ denotes the Heisenberg time associated with the mean level spacing $\delta_{E}=\left\langle E_{\ell+1}-E_{\ell}\right\rangle$ in the middle of the spectrum. Furthermore, $K_{\infty}=\sum_{\ell} f^{2}\left(E_{\ell}\right)$, where the value of $K_{\infty}$ is equivalently given by the inverse of the number $N_{\triangle E}$ of the eigenstates in the energy interval $\Delta E, K_{\infty} \approx N_{\Delta E}^{-1}$. As shown in Fig. 3, the RMT prediction agrees well with simulations for a finite-size chain in the limit of weak disorder (blue line) for times $\tau>\tau_{\mathrm{Th}}$, with $\tau_{\mathrm{Th}}$ the Thouless time defined as the onset of the ramp [38]. The flattening with increasing disorder strength (orange and green lines) is indicative of the crossover toward nonergodic (MBL) behavior.

The Hamiltonian (3) is time-reversal symmetric and thus its chaotic phase is described by the GOE. As discussed in Appendix C, this model can also be realized with complex $J$, so that the chaotic phase is described by a Gaussian unitary ensemble (GUE). In systems with broken timereversal symmetry, $K(\tau)$ follows the RMT prediction for the GUE,

$$
K_{\mathrm{GUE}}(\tau)=K_{\infty} \begin{cases}\frac{\tau}{\tau_{H}}, & 0<\tau \leq \tau_{H} \\ 1, & \tau>\tau_{H}\end{cases}
$$

Our SFF protocol described in the following exploits repeated QND measurements of the $c$ qubit both to prepare the desired initial energy distribution $f(E)$ in (10) [see Fig. 1(a)] as well as to read the SFF (10). The challenges faced in measuring the SFF and addressed in the following are the decoherence times in quantum simulators, relative to the times required to identify the "ramp," and the number of measurements required to provide clear signatures of both the ergodic and nonergodic regimes. Figure 3 plots simulated measurements for a finite measurement budget, which compare favorably with the (exact) numerical results for the SFF (solid lines). The question to be addressed is whether, with given experimental resources, it is possible to see the main characteristics of the SFF.

\section{B. Measurement protocol via QND-coupling to a $c$ qubit}

An experimental protocol to measure the SFF in the Hamiltonian case will require: (i) the ability to prepare an initial (microcanonical) ensemble of states $\rho_{\mathrm{mc}}=$ $\sum_{\ell} f\left(E_{\ell}\right)|\ell\rangle\langle\ell|$ with a filter function $f(E)$ of given width $\Delta E$ in the center of the spectrum; (ii) the ability to resolve, for a given number of measurements and thus signal-tonoise ratio (SNR), the baseline $K_{\infty} \approx N_{\Delta E}^{-1}$ and the value of the SFF around the Thouless time; and, finally, (iii) the ability to observe for a given decoherence time (part of) the $\operatorname{ramp} \tau_{\mathrm{Th}}<\tau<\tau_{H}$ and possibly the long-time behavior of $K(\tau)$.

\section{Preparation (verification) of a microcanonical ensemble}

The first step of the protocol requires preparation of the microcanonical (MC) ensemble $\rho_{\mathrm{mc}}=\sum_{\ell} f\left(E_{\ell}\right)|\ell\rangle\langle\ell|$. This can be achieved with a low-resolution phase estimation algorithm (PEA), providing a probabilistic preparation of an energy band via repeated measurement of the $c$ qubit. The PEA is based on the QND Hamiltonian (1) and requires a minimal number of measurements $M$ of the $c$ qubit to achieve a given measurement precision $\Delta E$ [33-35].

Figure 4 illustrates the idea of the preparation protocol. First, the many-body spin system initialized in a state $\rho_{\text {in }}$ and the $c$ qubit prepared in the state $|+\rangle_{c}$ are entangled by the QND interaction $\mathcal{H}_{\mathrm{QND}}(1)$ during a time $t_{0}$. As a result, the $c$ qubit is rotated proportionally to the values of the eigenenergies $E_{\ell}$ of the spin Hamiltonian (3). The time $t_{0}$ is chosen to maximally spread the full spectrum $E_{\ell}$ of the Hamiltonian $H_{\text {spin }}$ over the equator of the 


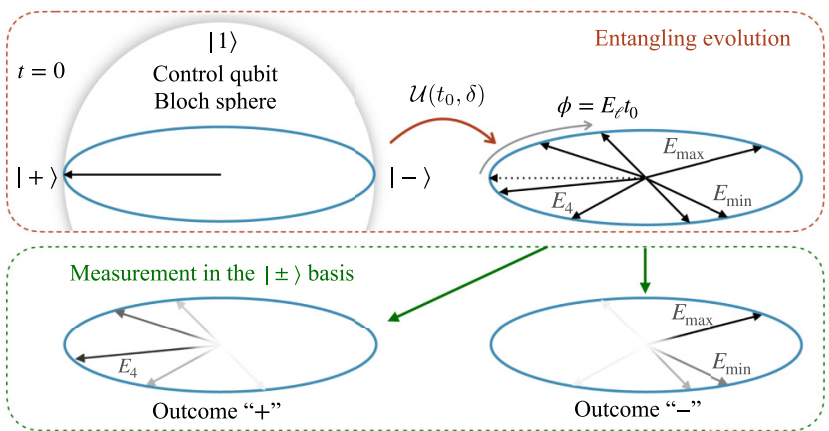

FIG. 4. The process of preparation of a microcanonical ensemble. The first filtering step with interaction time $t_{0}$ is shown. The QND interaction (1) rotates the $c$ qubit proportionally to the eigenenergy $E_{\ell}$ of the spin Hamiltonian (3). The measurement in the basis $| \pm\rangle$ leads to narrowing of the populated energy window of the spin state $\rho_{\text {out }}$. The shades of gray of the arrows corresponding to different $E_{\ell}$ represent the conditional probability for the eigenstate $|\ell\rangle$ to appear in $\rho_{\text {out }}$.

$c$-qubit Bloch sphere. After that, the $c$-qubit measurement in the $| \pm\rangle_{c}$ basis shrinks the populated energy window of the spin state by a factor of approximately 2 . Repeating the cycle with increasing interaction times $t_{m}$ and postselecting "+" outcomes results in the state $\rho_{\mathrm{mc}}$ with a narrow energy distribution.

Figure 1(a) shows the quantum circuit for the full preparation procedure involving $M=3 c$ qubits entangled with the simulator via

$$
\mathcal{U}\left(t_{m}, \delta\right)=\exp \left\{-i\left[\left(H_{\text {spin }}-\delta\right) \otimes|0\rangle_{c}\langle 0|\right] t_{m}\right\}
$$

Note that the same result can be achieved by performing sequential entanglement and measurement cycles with a single $c$ qubit. The interaction times are chosen as $t_{m} \equiv$ $2^{m} t_{0}, m=0, \ldots, M-1$ where $t_{0} \leq \pi /\left(\left|E_{\ell}-\delta\right|_{\max }\right)$. The renormalized detuning of the control laser $\delta$ introduced in Eq. (9) allows us to tune the energy band of the state to be prepared. If we now select a run with all readouts " $+_{m}$," $m=0, \ldots, M-1$, then the initial state of the spin system is projected into the state $\rho_{\text {out }}$ with a narrow distribution in the energy eigenbasis $|\ell\rangle$ (see Appendix E)

$$
\left\langle\ell\left|\rho_{\text {out }}\right| \ell\right\rangle \propto p_{\ell}=P\left(+_{0} \ldots+_{M-1}, E_{\ell}-\delta\right)\left\langle\ell\left|\rho_{\text {in }}\right| \ell\right\rangle,
$$

where

$$
P\left(+_{0} \ldots+_{M-1}, x\right)=\left\{\frac{\sin \left(2^{M} t_{0} x\right)}{2^{M} \sin \left(t_{0} x\right)}\right\}^{2} .
$$

The success probability of the preparation is $p_{\mathrm{mc}} \equiv \sum_{\ell} p_{\ell}$. The function (14) has a peak at $x=0$ with a width of approximately $2^{-M} / t_{0}$ and, therefore, the conditional outcome state exhibits an exponential narrowing of the energy distribution around $E_{\ell} \approx \delta$. Note, however, that

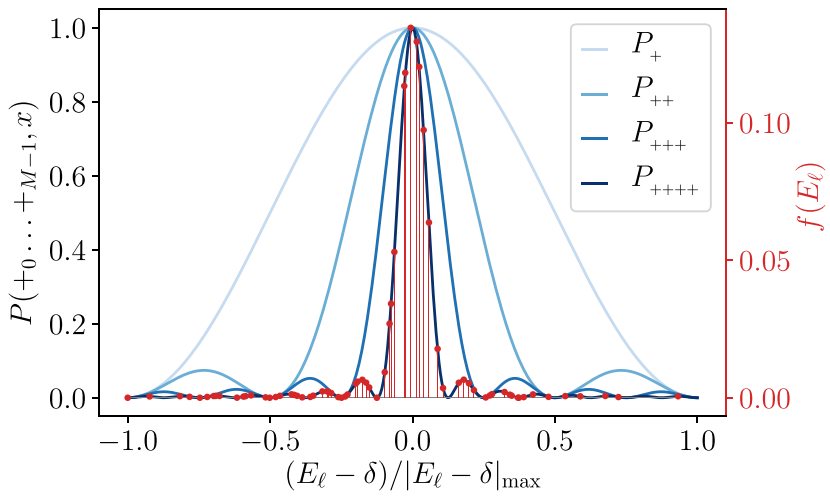

FIG. 5. The filter functions for $M=1,2,3,4$ filtering steps ( $c$ qubits) are shown in blue. The eigenstate probabilities $f\left(E_{\ell}\right)$ filtered with $M=4$ steps are shown in red.

the variance of this distribution has the same scaling of approximately $2^{-M} / t_{0}^{2}$ due to the presence of long tails.

Following the procedure described previously, the microcanonical ensemble can be prepared by initializing the spin system in the infinite-temperature state $\rho_{\text {in }}=\rho_{\infty} \propto \sum_{\ell}|\ell\rangle\langle\ell|$ and postselecting the outcome state with the probability $p_{\mathrm{mc}} \sim 2^{-M}$. As a result, the spin system will be probabilistically prepared in the microcanonical ensemble $\rho_{\mathrm{mc}}=\sum_{\ell} f\left(E_{\ell}\right)|\ell\rangle\langle\ell|$ with $f\left(E_{\ell}\right) \propto$ $\sum_{\ell} P\left(+_{0} \ldots+_{M-1}, E_{\ell}-\delta\right)$. The state has the mean energy $\bar{E}=\operatorname{Tr}\left\{H_{\text {spin }} \rho_{\mathrm{mc}}\right\}=\delta$ and the bandwidth $\Delta E=$ $\sqrt{\operatorname{Tr}\left\{\left(H_{\text {spin }}-\delta\right)^{2} \rho_{\mathrm{mc}}\right\}} \sim 2^{-M / 2} / t_{0}$. Note that the initial infinite-temperature ensemble $\rho_{\infty}$ can be sampled by random initialization of individual spins in up and down states, where we additionally apply the constraint $S_{z}=0$ (equal number of spins up and down) to probe the SFF in the zero-magnetization sector. An example of the resulting eigenstate probability distributions is shown in Fig. 5 for the Heisenberg chain (3) of $L=8$ spins.

We remark that measurement of the probability $p_{\mathrm{mc}}$ of the successful $\mathrm{MC}$ ensemble preparation allows us to estimate (for details, see Appendix $\mathrm{H}$ ) the Heisenberg time $\tau_{H}$ and the plateau value $K_{\infty}$ of the SFF as $\tau_{H} \simeq 2^{M+1} t_{0} \mathcal{D} p_{\mathrm{mc}}$ and $K_{\infty} \simeq(2 / 3)\left(\mathcal{D} p_{\mathrm{mc}}\right)^{-1}$. Here, $\mathcal{D}$ is the Hilbert-space dimension of the considered symmetry sector of the model. For the Heisenberg spin chain (3) with $S_{z}=0$, we have $\mathcal{D}=C_{L}^{L / 2} \approx \sqrt{2 / \pi L} 2^{L}$, where $C_{L}^{L / 2}$ is the binomial coefficient (we assume $L$ to be even). In the experiment, the probability $p_{\mathrm{mc}}$ is given by the ratio of the number of successful preparation attempts to the total number of experimental runs. The estimated values of $\tau_{H}$ and $K_{\infty}$ uniquely determine the behavior of the SFF in the chaotic regime assuming RMT (dashed lines in Figs. 3 and 6).

The low-resolution PEA procedure can also be used to verify that a given state consists of a superposition of energy eigenstates in a narrow energy interval $\Delta E$ around $\bar{E}$ (MC ensemble): with $\delta=\bar{E}$, the appearance 


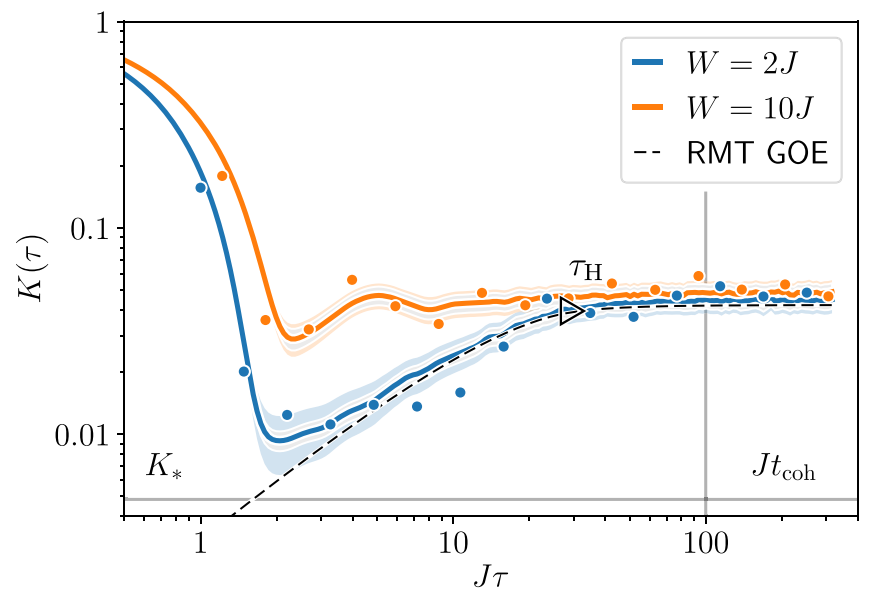

FIG. 6. Revealing the signatures of quantum chaos in the disordered Heisenberg chain of $L=8$ spins. The colored dots show the results of simulated measurements of $K(\tau)$ for two disorder strengths $W=2 J, 10 J$. The solid lines show the corresponding theoretical $K(\tau)$ and the black dashed line represents the RMT prediction $K_{\mathrm{GOE}}(\tau)$. The shaded areas show the rootmean-square error due to the shot noise and disorder averaging. The black triangle shows the Heisenberg time $\tau_{H}$. The model parameters are the same as in Fig. 3 (see also in the text).

of $M \simeq 2 \log _{2}\left(\left|E_{\ell}-\delta\right|_{\max } / \Delta E\right)$ successive "+" readouts with probability close to 1 signals that the given state has a desired energy variance. Further, if the state $\rho_{\text {in }}$ contains a collection of excited states in a narrow energy interval created from some initial state by a time-dependent perturbation (see, e.g., [40]), a MC state can be distilled with our procedure through several successive " + " readouts.

\section{Protocol to measure the SFF}

Following the preparation of the microcanonical ensemble $\rho_{\mathrm{mc}}$, we perform the evolution for a time $\tau$ with the QND-Hamiltonian (1) and finally measure the expectation values of $\sigma^{x}$ and $\sigma^{y}$ for the $c$ qubit, as shown in Fig. 1(a):

$$
\begin{aligned}
\left\langle\sigma^{x}(\tau)\right\rangle & =\operatorname{Tr}\left\{e^{-i \tau H_{\mathrm{spin}} \otimes|0\rangle_{c}(0 \mid} \rho_{\mathrm{mc}} \otimes|+\rangle_{c}\langle+| e^{i \tau H_{\mathrm{spin}} \otimes|0\rangle_{c}(0 \mid} \sigma^{x}\right\} \\
& =\sum_{\ell} f\left(E_{\ell}\right) \cos \left(E_{\ell} \tau\right), \\
\left\langle\sigma^{y}(\tau)\right\rangle & =\sum_{\ell} f\left(E_{\ell}\right) \sin \left(E_{\ell} \tau\right) .
\end{aligned}
$$

These provide us with $\left|\sum_{\ell} f\left(E_{\ell}\right) e^{-i E_{\ell} \tau}\right|^{2} \approx\left\langle\sigma^{x}(\tau)\right\rangle^{2}+$ $\left\langle\sigma^{y}(\tau)\right\rangle^{2}$ (see Appendix F). We obtain $K(\tau)$ by repeating this sequence for different disorder realizations and averaging the result.

The SFF measurement scheme realizes a QND measurement, meaning that the initial state of the spin system is not heated up or destroyed after the interaction with the $c$ qubit. It is, therefore, possible to reuse the once-prepared $\rho_{\mathrm{mc}}$ for the measurement of $K(\tau)$ with different times $\tau_{i}$ (see
Appendix G). The maximum number of recycling times $N_{\text {reuse }}$ is restricted by the coherence time $t_{\text {coh }}$ of the spin system as $\sum_{i=1}^{N_{\text {reuse }}} \tau_{i} \ll t_{\text {coh }}$.

\section{Experimental challenges}

An experimental realization of the SFF measurement faces two main challenges, which limit the achievable system sizes.

Time scales. Propagation up to the Heisenberg time $\tau_{H}$, which grows exponentially with the system size $L$, is limited by the finite coherence time of the quantum simulator. Thus, observation of the behavior of $K(\tau)$ at times $\tau \sim \tau_{\mathrm{H}}$ requires coherence times $t_{\text {coh }}>\tau_{\mathrm{H}} \sim 2^{L} /(J L)$. We note, however, that the effects of interest, such as the transition to chaotic dynamics at the Thouless time $\tau_{\mathrm{Th}}$ and the distinct behaviors of the SFF for quantum chaotic $[K(\tau) \sim \tau]$ and integrable systems $[K(\tau) \sim$ const.], take place at much shorter times in comparison with $t_{\text {coh }}$ (see Appendix D).

Signal magnitude. The second challenge is the exponentially small values of the SFF $\sim 2^{-L}$ at the characteristic times. The threshold signal level, which can be distinguished from the shot noise after averaging over $N_{\mathrm{d}}$ realizations of disorder in the spin Hamiltonian and $N$ measurements per one disorder realization, is given by $K_{*} \equiv 2(1+\sqrt{2}) /\left(N \sqrt{N_{\mathrm{d}}}\right)$ (see Appendix F). Thus, the total number of experimental runs per data point necessary to resolve the features of interest in $K(\tau)$ is given by $N_{\text {run }}>2^{L} \sqrt{N_{\mathrm{d}}} / N_{\text {reuse }}$ for the probabilistic preparation of the initial MC ensemble (see Appendix D).

\section{Numerical simulation of the SFF measurement}

To demonstrate the feasibility of the SFF measurement with our protocol, we perform numerical simulations of the measurement process in the disordered Heisenberg spin chain for various numbers $L$ of spins. The simulation includes the probabilistic preparation of the initial state $\rho_{\mathrm{mc}}$ and averaging over finite numbers of disorder realizations and experimental runs.

The results are presented in Figs. 3 and 6. According to Fig. 6, a small system of $L=8$ spins with a measurement budget of $N_{\text {run }} \simeq 10^{4}$ experimental runs per data point allows identification of the linear ramp in $K(\tau)$. This indicates the chaotic behavior with level repulsion in the Heisenberg model with a weak disorder $W=2 \mathrm{~J}$ (blue dots). In contrast, a strong disorder $W=10 J$ results in the localized behavior of the system dynamics (orange dots) lacking correlations in the distribution of the eigenenergies. The colored lines and the corresponding shaded areas represent the numerical prediction for $K(\tau)$ and the root-mean-square error of the simulated measurement, respectively. The black dashed line is the RMT prediction $K_{\mathrm{GOE}}(\tau)$ Eq. (12).

The Thouless times $\tau_{\mathrm{Th}}$ for various disorder strengths can be probed in a larger system of $L=12$ spins, as shown 
in Fig. 3. It is evident from the figure that the time $\tau$ at which the data points approach the RMT prediction $K_{\mathrm{GOE}}(\tau)$ given by the black dashed line grows with the increase of the disorder strength $W$, which is compatible with the expected behavior of the Thouless time $\tau_{\mathrm{Th}}$. The root-mean-square error of the simulated measurement with $N_{\text {run }} \simeq 2 \times 10^{5}$ experimental runs per data point is shown with shaded areas around the solid lines indicating the numerical prediction for $K(\tau)$.

In both Figs. 3 and 6 , the horizontal and vertical gray lines mark the shot-noise threshold $K_{*}$ and the coherence time $t_{\text {coh }} \sim 10^{2} J^{-1}$ in our Rydberg setup (see Appendix D), respectively. For the system of $L=8(12)$ spins, we simulate $\mathrm{MC}$ ensemble preparation with $M=$ 3 (5) filtering steps, perform averaging over $N_{\mathrm{d}}=100$ (20), and include recycling of the prepared $\mathrm{MC}$ ensemble for $N_{\text {reuse }}=10$ times. The parameters of the Heisenberg model (3) for both system sizes are $\Delta=0.8, J_{2}=0.02$, and $\Delta_{2}=$ 0.06 .

It is important to stress that in both cases of $L=8$ and $L=12$, the $K_{\mathrm{GOE}}(\tau)$ curves (black dashed lines) are completely determined by the plateau value $K_{\infty}$ for $\tau \rightarrow \infty$ and by the Heisenberg time $\tau_{H}$, both of which can be independently estimated as outlined in Sec. III B1 and discussed in Appendix H.

\section{MEASUREMENT OF THE SFF IN FLOQUET SYSTEMS}

To study aspects of ergodicity, thermalization, and quantum chaotic dynamics, periodically driven or Floquet systems are particularly appealing for several reasons. First, due to the absence of energy conservation, generic Floquet systems can thermalize very rapidly and completely even for relatively small system sizes [63]. Further, since the density of quasienergies is generically flat, it is not necessary to unfold the spectra of Floquet systems to access their spectral statistics [58]. This relative simplicity of Floquet systems has led to intriguing recent results that, through explicit calculations of the SFF, establish an analytical connection between RMT and many-body quantum chaos in interacting periodically driven spin chains $[37,64-66]$. As we discuss in the following, such Floquet spin models, and the experimental measurement of the SFF, can be realized naturally with Rydberg-dressing schemes.

\section{A. SFF of Floquet systems}

In Floquet systems, we are interested in the statistics of eigenvalues of the unitary operator $U(\theta)$, which describes the evolution of the system during one driving period of duration $\theta: U(\theta)|\ell\rangle=e^{-i \lambda_{\ell} \theta}|\ell\rangle$, where $\lambda_{l} \in[0,2 \pi / \theta]$ is the quasienergy. Similar to the Hamiltonian case [see Eq.
(10)], we define the SFF for Floquet systems as [64]

$$
K(t)=\overline{\left|\sum_{\ell} f_{l} e^{-i \lambda_{\ell} \theta t}\right|^{2}}=\overline{\left|\operatorname{tr}\left[U(\theta)^{t} \rho_{\text {in }}\right]\right|^{2}},
$$

where the integer "time" $t$ is the number of the evolution periods, $\rho_{\text {in }}=\sum_{\ell} f_{l}|\ell\rangle\langle\ell|$ is the initial quantum state, and the overline represents a possible average over disorder. The flat density of states of quantum chaotic Floquet systems allows us to use the infinite-temperature ensemble $\rho_{\text {in }}=\rho_{\infty}=\mathcal{D}^{-1} \sum_{\ell}|\ell\rangle\langle\ell| \propto \mathbb{I}$ as the initial state (here, $\mathcal{D}$ is the dimension of the Hilbert space).

The behavior of the SFF in Floquet dynamics has the same characteristic features as in the Hamiltonian case. As an illustration, in the following we consider two Floquet systems. First, we present a Floquet model that exhibits a crossover between the circular orthogonal ensemble (COE) and the circular unitary ensemble (CUE) as a function of the driving frequency $\omega=2 \pi / \theta$ [67]. Then we consider kicked Ising models that demonstrate clear signatures of COE and CUE statistics even for a small system of $L=4$ spins.

Crossover between COE and CUE statistics. Here, we consider an interesting example of a periodically driven system for which a random matrix class of the Floquet operator $U(\theta)$ differs from that of the time-dependent Hamiltonian $H(t)$ that generates the dynamics of the system [67]. To be specific, we consider a piecewise constant Hamiltonian with $H_{1}$ during the first half of the driving period and $\mathrm{H}_{2}$ during the second half. The evolution operator over one period is of the form

$$
U(\theta)=e^{-i(\theta / 2) H_{1}} e^{-i(\theta / 2) H_{2}},
$$

where $H_{1}$ and $H_{2}$ are Heisenberg Hamiltonians with random magnetic fields $h_{i}^{x, y, z}$, which are normally distributed around zero with unit variance:

$$
\begin{aligned}
& H_{1}=J \sum_{i}^{L}\left\{\sum_{\eta=x, y, z} \sigma_{i}^{\eta} \sigma_{i+1}^{\eta}+\frac{1}{2}\left(h_{i}^{x} \sigma_{i}^{x}+h_{i}^{y} \sigma_{i}^{y}\right)\right\}, \\
& H_{2}=J \sum_{i}^{L}\left\{\sum_{\eta=x, y, z} \sigma_{i}^{\eta} \sigma_{i+1}^{\eta}+\frac{1}{2}\left(h_{i}^{z} \sigma_{i}^{z}-h_{i}^{y} \sigma_{i}^{y}\right)\right\} .
\end{aligned}
$$

The Heisenberg models can be realized in the quantum simulator based on dressed Rydberg atoms as discussed in Sec. II. For generic values of the driving period $\theta$, the spectral statistics of the Floquet operator $U(\theta)$ are described by the CUE. However, for finite system sizes and in the limit of high driving frequencies, the dynamics of the system are described by an effective time-independent Hamiltonian $\left(H_{1}+H_{2}\right) / 2$ in which the random fields in the $y$ direction cancel and time-reversal symmetry is restored. Therefore, in this limit, the spectral statistics of $U(\theta)$ 


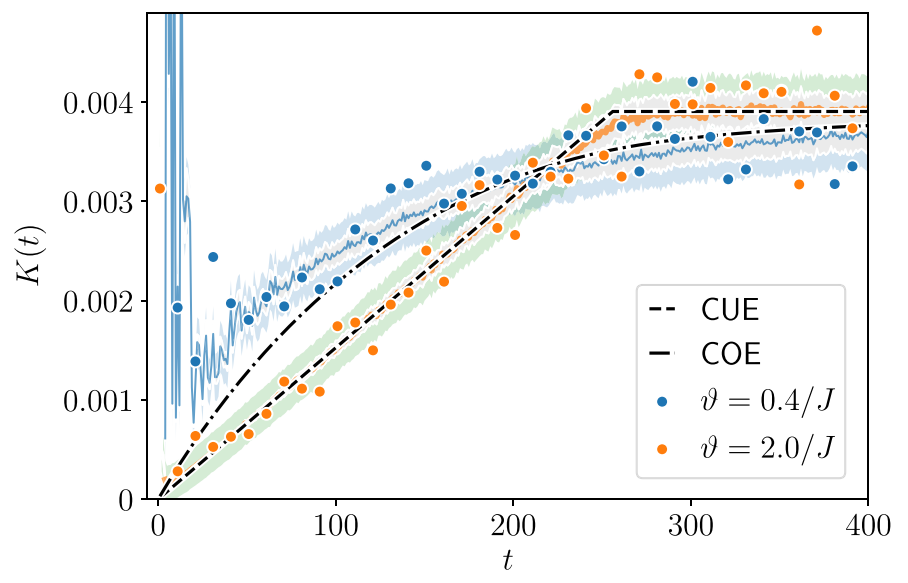

FIG. 7. The spectral form factor in the disordered Floquet model given in Eq. (16). The model exhibits a crossover between COE (blue dots) and CUE (orange dots) statistics as a function of the driving period $\theta$. The black dashed and dot-dashed lines show the RMT predictions $K_{\mathrm{CUE}}(t)$ and $K_{\mathrm{COE}}(t)$, respectively. The numerical simulations are performed for $L=8$ spins with $5 \times 10^{5}$ measurements per data point.

belong to the COE. We note that in this limit, the density of quasienergies is determined by the effective Hamiltonian and is not flat. However, in the numerical examples that follow, we find that effects due to a nonflat density of quasienergies are insignificant and we take $\rho_{\text {in }}$ in Eq. (15) to also be the infinite-temperature ensemble for high driving frequencies.

The SFF and the simulated measurement of the SFF (see Sec. IV B) are shown in Fig. (7). Remarkably, even the relatively small Floquet system of $L=8$ spins exhibits clear RMT behavior with a crossover between COE and CUE statistics upon changing the Floquet period $\theta$. In particular, we observe COE statistics for short periods or high driving frequencies (blue dots) with $\theta<\theta_{c} \sim 0.5 / J$. The dot-dashed line shows the corresponding RMT prediction $K_{\mathrm{COE}}(t)=[2 t-t \ln (1+2 t / \mathcal{D})] / \mathcal{D}^{2}$ for $0<t<\mathcal{D}$, where the Heisenberg time is set by the Hilbert-space dimension $\mathcal{D}=2^{L}$ [see Eq. (12)]. For long periods $\theta>$ $\theta_{c}$, the system is in the unitary class with CUE statistics (orange dots) resulting in $K_{\mathrm{CUE}}(t)=t / \mathcal{D}^{2}$ for $0<t<\mathcal{D}$ (dashed line) [see also Eq. (13)]. We note that for sufficiently large values of $\theta$, the initial decay due to dephasing lasts at most a few driving cycles. This is because for these values of $\theta$, the unitary $U(\theta)$ is not a sparse matrix, as it is for $\theta \ll 1$, but a dense one coupling practically all states in the Hilbert space with each other. As a result, chaotic behavior starts already at $t=t_{\mathrm{Th}} \sim 1$.

Small Floquet systems. We now consider two kicked Ising models described by the evolution operators

$$
U_{2}(\theta)=e^{-i H_{x} \theta} e^{-i H_{y} \theta}
$$

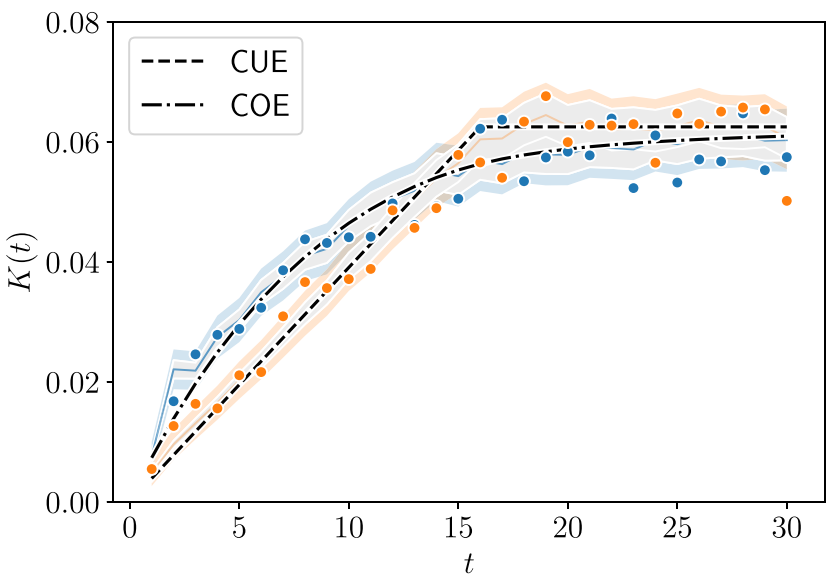

FIG. 8. The spectral form factor in the disordered kicked Ising models Eqs. (17) and (18). Already in the small system of $L=4$ spins, the models exhibit a clear distinction between the statistics of the $\operatorname{COE}\left[U_{2}(\theta)\right.$ model shown with blue dots] and the CUE $\left[U_{3}(\theta)\right.$ model shown with orange dots]. The black dashed and dot-dashed lines show the RMT predictions $K_{\mathrm{CUE}}(t)$ and $K_{\mathrm{COE}}(t)$, respectively. The numerical simulations are performed for $3.6 \times$ $10^{4}$ measurements per data point with driving period $\theta=1 / \mathrm{J}$.

$$
U_{3}(\theta)=e^{-i H_{x} \theta} e^{-i H_{y} \theta} e^{-i H_{z} \theta},
$$

where $H_{x, y, z}$ are the transverse Ising Hamiltonians with random magnetic fields $h_{i}^{x, y, z} \in[-1,1]$ :

$$
\begin{aligned}
& H_{x}=J \sum_{i}^{L}\left(\sigma_{i}^{x} \sigma_{i+1}^{x}+h_{i}^{y} \sigma_{i}^{y}\right), \\
& H_{y}=J \sum_{i}^{L}\left(\sigma_{i}^{y} \sigma_{i+1}^{y}+h_{i}^{z} \sigma_{i}^{z}\right), \\
& H_{z}=J \sum_{i}^{L}\left(\sigma_{i}^{z} \sigma_{i+1}^{z}+h_{i}^{x} \sigma_{i}^{x}\right) .
\end{aligned}
$$

The two models described by the Floquet operators $U_{2}(\theta)$ and $U_{3}(\theta)$ belong to the COE and CUE random matrix classes, respectively. Remarkably, the statistical distinction can already be seen in a small system of $L=4$ spins, as shown by the simulated measurement of the SFF in Fig. 8. In the quantum simulator based on Rydberg atoms, the Ising models can be realized according to the general scheme presented in Sec. II.

These examples illustrate that, as in the case of the Hamiltonian systems, the SFF provides a sensitive tool for probing quantum chaotic behavior of Floquet systems. At the same time, the measurement of the SFF in Floquet systems faces the same challenges as are present in Hamiltonian systems, i.e., small values of the signal and exponentially long time scales. However, for experimental studies of many-body quantum chaos, Floquet systems can be beneficial because they typically exhibit pronounced 
RMT behavior even for comparatively small system sizes. Moreover, since spectral filtering is not required in Floquet systems, the preparation step described in Sec. III B 1 can be omitted.

\section{B. Measurement protocol}

To generalize the measurement protocol for the SFF in Hamiltonian systems described in Sec. III to Floquet systems, we reformulate it in terms of the unitary operator $\mathcal{U}(t)$, which describes the coupled evolution of the system and the $c$ qubit during $t$ Floquet periods of duration $\theta$ :

$$
\mathcal{U}(t)=U(\theta)^{t} \otimes|0\rangle_{c}\langle 0|+\mathbb{I} \otimes| 1\rangle_{c}\langle 1| .
$$

For simplicity, we focus here on Floquet systems with piecewise constant Hamiltonians $H_{k}$ for time periods $\tau_{k-1}<\tau<\tau_{k}$, where $\tau_{0}=0$ and the time dependence is repeated periodically with period $\theta=\sum_{k} \tau_{k}$. The corresponding Floquet operator reads $U(\theta)=\prod_{k} e^{-i \tau_{k} H_{k}}$. The controlled evolution given in Eq. (19) is achieved by using the QND interaction Hamiltonian (1) between the spin system and the $c$ qubit with $H_{\text {spin }} \equiv H_{k}$ :

$$
\mathcal{U}(t)=\left[\prod_{k} e^{-i \tau_{k} H_{k} \otimes|0\rangle_{c}\langle 0|}\right]^{t} .
$$

The measurement protocol for the SFF starts with the initialization of the $c$ qubit in the state $|+\rangle$ and the system spins in the infinite-temperature state $\rho_{\infty}$. (In practice, it is sufficient to sample from the infinite-temperature ensemble by preparing the system, e.g., in random product states.) Then, for a particular realization of disorder in the instantaneous Hamiltonians $H_{k}$, we apply the controlled evolution $\mathcal{U}(t)$ and measure the expectation values of the operators $\sigma_{x}$ and $\sigma_{y}$ for the $c$ qubit afterward:

$$
\begin{aligned}
\left\langle\sigma^{x}(t)\right\rangle & =\operatorname{Tr}\left\{\mathcal{U}(t) \rho_{\infty} \otimes|+\rangle_{c}\langle+| \mathcal{U}(t)^{\dagger} \sigma^{x}\right\}, \\
& =\operatorname{Re}\left\langle U(\theta)^{t}\right\rangle \\
\left\langle\sigma^{y}(t)\right\rangle & =\operatorname{Im}\left\langle U(\theta)^{t}\right\rangle .
\end{aligned}
$$

Finally, the quantity $\left|\left\langle U(\theta)^{t}\right\rangle\right|^{2}=\left\langle\sigma^{x}(t)\right\rangle^{2}+\left\langle\sigma^{y}(t)\right\rangle^{2}$ is averaged over disorder realizations, resulting in the SFF $K(t)$ for the Floquet system given in Eq. (15).

Experimental limitations of the measurement of the SFF in Floquet systems are analogous to the Hamiltonian case, as discussed in Sec. III B2. As an example, the effect of a finite number of measurements is illustrated in Figs. 7 and 8 for the Floquet systems described by Eqs. (16)-(18), respectively.

\section{CONCLUSIONS AND OUTLOOK}

Recent experimental studies of ergodicity breaking in quantum many-body systems focus on the absence of thermalization of local observables in integrable [68-70] and many-body localized systems [71-74], and on the slow growth of entanglement [74-76]. While it is firmly established through theoretical work that key signatures of ergodic versus nonergodic dynamics are carried by individual eigenstates of the Hamiltonian of a quantum many-body system and the statistics of the corresponding eigenvalues, accessing these signatures in experiments requires approaches beyond the standard paradigm of quantum simulation. As a first main result of the present work, we develop a method that enables in-depth experimental studies of the level statistics of interacting quantum many-body systems through the measurement of the SSF and we discuss the feasibility of observing the SFF signatures of ergodic (RMT) versus nonergodic dynamics for both disordered 1D Heisenberg and Floquet-spin models. We conclude that the key features of RMT in the SFF should be observable for system sizes of ten or more atoms based on techniques that are available in present or will be available in next-generation Rydberg experiments. We also emphasize that experimental realization will strongly benefit from the ongoing development to improve coherence times and implement high-fidelity (nondestructive) readout of the Rydberg qubits, which are aimed at advancing scalable quantum computing on the Rydberg platform.

In a broader context, our method to measure the SFF is an example of a quantum protocol in which the state of a quantum simulator is prepared and monitored via the measurement of an auxiliary qubit, which is entangled with the quantum simulator by applying a QND gate. This is the second main result of our work: the implementation of a QND Hamiltonian $\mathcal{H}_{\mathrm{QND}}=H_{\text {spin }} \otimes|0\rangle_{c}\langle 0|$ with Rydberg-tweezer arrays, which yields a QND gate as $\mathcal{U}_{\mathrm{QND}}(t)=e^{-i \mathcal{H}_{\mathrm{QND}} t}$. In the context of a many-body system engineered with a Rydberg-tweezer platform, this QND Hamiltonian can be implemented for a broad class of spin models specified by a Hamiltonian $H_{\text {spin }}$. While we consider 1D systems in form of a ring with the $c$ qubit in the center, our ideas also carry over to more complex 2D simulator geometries $[1,4,43]$. In addition, unique opportunities to combine quantum simulation with atomic clocks are offered by alkaline-earth atoms $[3,18,77,78]$.

An intriguing possibility opened up by the present study is the design and implementation of more general quantum protocols involving QND gates entangling the quantum simulator with a freely designable $H_{\text {spin }}$ with a set of $c$ qubits. As noted before, this opens the door to running, for example, quantum phase estimation algorithms on analog quantum simulators. In Ref. [79], a continuous readout of the "energy" of a quantum many-body system has been proposed as the analog measurement of a homodyne current, with an implementation for a transverse Ising model with long-range interaction. In contrast, quantum phase estimation based on the present QND gate with a freely designable $H_{\text {spin }}$ provides an essentially universal 
digital quantum algorithm to achieve measurement and preparation of (a narrow band of) energy eigenstates in an analog quantum simulator setting. Moreover, quantum phase estimation can be utilized to compute the dynamical response functions of quantum many-body systems [80]. This opportunity is of particular interest in the context of NMR, where the ability to design $H_{\text {spin }}$ enables access to the NMR spectra of molecules that are described by parametric Heisenberg models [81].

Note added. After submission of the present work, we have become aware of Ref. [56] by Young et al., which proposes to realize multiqubit Rydberg-blockade gates using microwave-dressed Rydberg states.

\section{ACKNOWLEDGMENTS}

We thank M. Lukin for helpful discussions. This work is supported by the European Union's Horizon 2020 research and innovation programme under Grant Agreements No. 817482 (Programmable Atomic Large-Scale Quantum Simulation, PASQuanS) and No. 731473 [Quantum Technologies European Research Area Network (QuantERA) via Quantum Technologies For LAttice Gauge theories (QTFLAG)], the U.S. Air Force Office of Scientific Research (AFOSR) via IOE Grant No. FA9550-19-1-7044 LASCEM, by the Simons Collaboration on Ultra-Quantum Matter, which is a grant from the Simons Foundation (Grant No. 651440, P.Z.), and by the Institut für Quanteninformation. The parameters for the Rydberg simulations were obtained using the Alkali Rydberg Calculator (ARC) library [82].

D. V. Vasilyev and A. Grankin contributed equally to this work.

\section{APPENDIX A: DIPOLE-DIPOLE INTERACTIONS}

In this appendix, we provide details on the van der Waals interactions between $n P_{1 / 2}+n P_{1 / 2}$ and $n P_{1 / 2}+$ $n^{\prime} S_{1 / 2}$ states as relevant for the model of Sec. II. Our discussion adapts and extends Ref. [43].

For any pair of atoms $i$ and $j$, the dipole-dipole interaction Hamiltonian reads [46]

$$
V_{\mathrm{dd}}^{(i, j)}\left(\vec{r}_{i j}\right)=\vec{d}^{(i)} \vec{d}^{(j)} / r_{i j}^{3}-3\left(\vec{d}^{(i)} \vec{r}_{i j}\right)\left(\vec{d}^{(j)} \vec{r}_{i j}\right) / r_{i j}^{5},
$$

where $\vec{d}^{(i)}$ is the dipole operator of the $i$ th atom and $\vec{r}_{i j}$ is the relative distance between atoms. In second-order perturbation theory in $\hat{V}_{\mathrm{dd}}^{(i, j)}$, we obtain the effective van der Waals interaction $[43,83]$ (we assume the absence of Förster resonances[48]):

$$
H_{\mathrm{vdW}}^{(i, j)} \equiv P \sum_{\beta, \chi} \frac{V_{d d}^{(i, j)} Q_{\beta \chi} V_{d d}^{(i, j)}}{\delta_{\beta \chi}} P,
$$

Here, $\hat{P}$ is the projector onto the states of interest $\left(n P_{1 / 2}, n P_{1 / 2}\right.$ or $\left.n P_{1 / 2}, n^{\prime} S_{1 / 2}\right)$ and $Q_{\beta \chi} \equiv|\beta, \chi\rangle\langle\beta, \chi|$ is the projector on manifolds that are populated only as virtual intermediate states, with $\delta_{\beta \chi}$ energy differences. We note that due to the perturbative nature of (A2), this expression is valid only beyond a certain critical radius $r>r_{c}$ (see the following).

\section{Interaction between simulator atoms}

We first consider the van der Waals interaction between excited Rydberg states for the fine-structure states $\left|r_{\alpha= \pm}\right\rangle=\left|n P_{1 / 2}, m_{J}= \pm 1 / 2\right\rangle$ of simulator atoms. The relevant projector reads

$$
P=\sum_{\alpha= \pm}\left|r_{\alpha}\right\rangle_{i}\left\langle r_{\alpha}\left|\otimes \sum_{\alpha= \pm}\right| r_{\alpha}\right\rangle_{j}\left\langle r_{\alpha}\right|
$$

The possible interaction channels for the atoms in $P_{1 / 2}$ states are listed in Table $\mathrm{I}$, which provides us with the intermediate states $|\beta, \chi\rangle$ of Eq. (A2). This allows us to write the matrix of the van der Waals interaction Hamiltonian $H_{\mathrm{vdW}}^{(i, j)}$ in the form of Eq. (7) [57], with $C_{6}=2\left[C_{6}^{(a)}+4 C_{6}^{(b)}+2\left(C_{6}^{(c)}+C_{6}^{(d)}\right)\right] / 27$ and $\widetilde{C}_{6}=C_{6}^{(a)}+C_{6}^{(b)}-C_{6}^{(c)}-C_{6}^{(d)}$ combinations of coefficients $C_{6}^{(a, b, c, d)}$ attributed to different scattering channels. (Explicit expressions involving Clebsch-Gordan and dipole matrix elements can be found in Ref. [43,83]) and corresponding plots for $\mathrm{Rb}$ atoms are shown in Fig. 9(a). The $4 \times 4$ matrix $\mathbb{D}_{0}(\theta, \phi)$ referred to in Eq. (7) reads

$$
\begin{aligned}
& \mathbb{D}_{0}(\theta, \phi)= \\
& \left(\begin{array}{ccccc}
\frac{3 \cos (2 \theta)-1}{81} & \frac{4 e^{-i \phi} \sin (2 \theta)}{27} & \frac{4 e^{-i \phi} \sin (2 \theta)}{27} & \frac{2 e^{-2 i \phi} \sin ^{2}(\theta)}{27} \\
\frac{e^{i \phi} \sin (2 \theta)}{27} & \frac{1-3 \cos (2 \theta)}{81} & \frac{-5-3 \cos (2 \theta)}{81} & \frac{-4 e^{-i \phi} \sin (2 \theta)}{27} \\
\frac{e^{i \phi} \sin (2 \theta)}{27} & \frac{-5-3 \cos (2 \theta)}{81} & \frac{1-3 \cos (2 \theta)}{81} & \frac{-4 e^{-i \phi} \sin (2 \theta)}{27} \\
\frac{2 e^{2 i \phi} \sin ^{2}(\theta)}{27} & \frac{-4 e^{i \phi} \sin (2 \theta)}{27} & \frac{-4 e^{i \phi} \sin (2 \theta)}{27} & \frac{3 \cos (2 \theta)-1}{81}
\end{array}\right)
\end{aligned}
$$


TABLE I. The channels of the dipole-dipole interaction of $n P_{1 / 2}$ Rydberg states.

\begin{tabular}{llll}
\hline \hline (a) & $n P_{1 / 2}+n P_{1 / 2}$ & $\leftrightarrow$ & $n_{\beta} S_{1 / 2}+n_{\chi} S_{1 / 2}$ \\
\hline (b) & $n P_{1 / 2}+n P_{1 / 2}$ & $\leftrightarrow$ & $n_{\beta} D_{3 / 2}+n_{\chi} D_{3 / 2}$ \\
(c) & $n P_{1 / 2}+n P_{1 / 2}$ & $\leftrightarrow$ & $n_{\beta} D_{3 / 2}+n_{\chi} S_{1 / 2}$ \\
(d) & $n P_{1 / 2}+n P_{1 / 2}$ & $\leftrightarrow$ & $n_{\beta} S_{1 / 2}+n_{\chi} D_{3 / 2}$ \\
\hline \hline
\end{tabular}

and following the geometry of our setup in Fig. 1(b), we set $\theta=\pi / 2$. Thus we obtain, for the interaction Hamiltonian, the structure

$$
V_{\mathrm{vdW}}^{(i, j)}=\left(\begin{array}{cccc}
W_{++}^{(i, j)} & 0 & 0 & V_{++}^{(i, j)} \\
0 & W_{+-}^{(i, j)} & V_{-+}^{(i, j)} & 0 \\
0 & V_{+-}^{(i, j)} & W_{-+}^{(i, j)} & 0 \\
V_{--}^{(i, j)} & 0 & 0 & W_{--}^{(i, j)}
\end{array}\right),
$$

where

$$
\begin{aligned}
& W_{++}^{(i, j)}=W_{--}^{(i, j)}=\frac{1}{r_{i j}^{6}}\left[C_{6}-\frac{4}{81} \widetilde{C}_{6}\right], \\
& W_{+-}^{(i, j)}=W_{-+}^{(i, j)}=\frac{1}{r_{i j}^{6}}\left[C_{6}+\frac{4}{81} \widetilde{C}_{6}\right], \\
& V_{+-}^{(i, j)}=V_{-+}^{(i, j)}=-\frac{2}{81} \frac{\widetilde{C}_{6}}{r_{i j}^{6}},
\end{aligned}
$$

and

$$
V_{++}^{(i, j)}=V_{--}^{(i, j) *}=-\frac{2}{27} \frac{\widetilde{C}_{6}}{r_{i j}^{6}} \exp (-2 i \phi)
$$

The specific spin models that can be engineered via Rydberg dressing [43,44], i.e., by admixing the van der Waals interactions (A3) to the ground states by off-resonant laser light, are determined by the structure of the matrix elements given in Eq. (A4).

\section{Interactions between simulator and control atoms}

Here, we consider the van der Waals interaction between the simulator and control atoms in the Rydberg states. We restrict the states of the control atom to the Zeeman manifold, which includes the logical qubit state $|1\rangle_{c}$, i.e., $|1\rangle_{c}=$ $\left|n^{\prime} S_{1 / 2}, m_{j}=1 / 2\right\rangle$, and $\left|1^{\prime}\right\rangle_{c} \equiv\left|n^{\prime} S_{1 / 2}, m_{j}=-1 / 2\right\rangle$. As we discuss in the following, the unwanted coupling between $|1\rangle_{c}$ and $\left|1^{\prime}\right\rangle_{c}$ can be minimized by proper choice of the principal quantum number $n^{\prime}$ [57]. For the states of the simulator atoms, we again consider $\left|r_{\alpha= \pm}\right\rangle=$ $\left|n P_{1 / 2}, m_{J}= \pm 1 / 2\right\rangle$. As already mentioned in the main text, we assume that $\left|n-n^{\prime}\right| \gg 1$ in order to avoid direct dipolar exchange interactions between the control and simulator atoms. A comparison of the typical values of the (a)

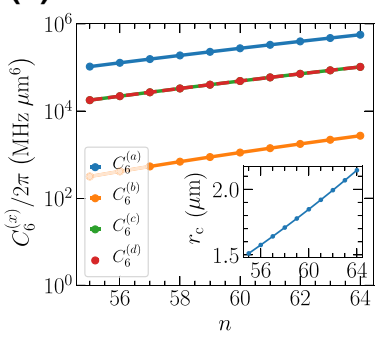

(b)

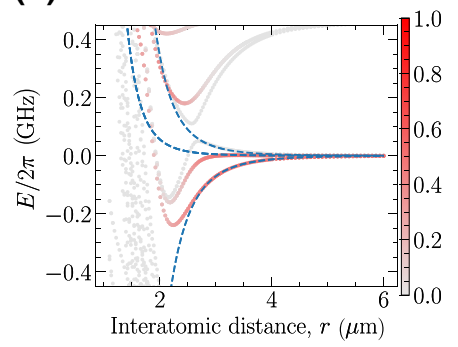

FIG. 9. The van der Waals interaction of two $n P_{1 / 2}$ Rydberg states. (a) The $C_{6}^{(x)}$ coefficient as a function of the principal quantum number $n$. The inset shows the critical radius $r_{c}$. (b) The exact diagonalization of the dipole-dipole interaction given in Eq. (A1). The colors indicate the projection of exact eigenstates onto the state $\left|r_{+}\right\rangle_{i}\left|r_{+}\right\rangle_{j}$. The blue dashed line denotes the eigenvalues of the perturbative Hamiltonian given in Eq. (A3).

van der Waals and dipolar interaction strengths is shown in Fig. 10(a) for distances $R_{\max } \approx 4.6 \mu \mathrm{m}$.

The relevant scattering channels are listed in Table II. Similar to (A 1), we obtain for $H_{\mathrm{vdW}}^{(i, c)}$ in the basis $\left(\left|r_{+}\right\rangle|1\rangle_{c}\right.$, $\left.\left|r_{+}\right\rangle\left|1^{\prime}\right\rangle_{c},\left|r_{-}\right\rangle|1\rangle_{c},\left|r_{-}\right\rangle\left|1^{\prime}\right\rangle_{c}\right)$ an expression analogous to Eq. (7),

$$
V_{\mathrm{vdW}}^{(i, c)}=\frac{1}{r_{i, c}^{6}}\left[C_{6}^{\prime} \mathbb{I}_{4}-\widetilde{C}_{6}^{\prime} \mathbb{D}_{0}(\theta, \phi)\right]
$$

where the coefficients $C_{6}^{\prime}$ and $\widetilde{C}_{6}^{\prime}$ are defined as previously. The numerical results for $C_{6}^{\prime}$ and the relative strength of the second (anisotropic) term in Eq. (A5) are shown in Figs. 11(a) and 11(b), respectively, for different principal quantum numbers $n$ and $n^{\prime}=n+\Delta n$. As can be seen from Fig. 11(b), the second term in $V_{\mathrm{vdW}}^{(i, c)}$ can be made much (a)

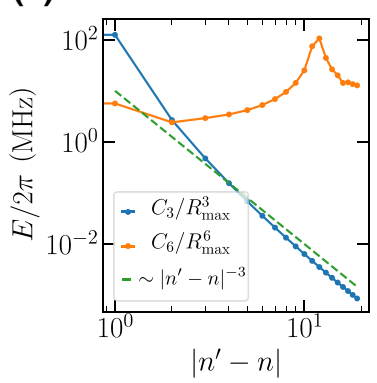

(b)

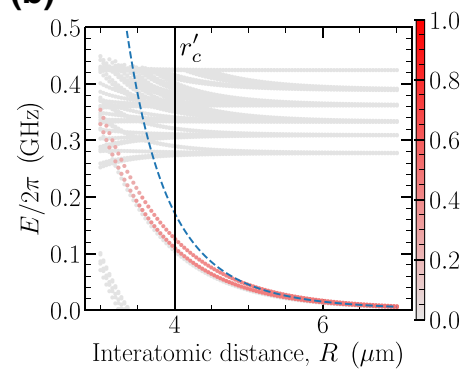

FIG. 10. The interaction energy between the control and simulator atoms in the Rydberg states. (a) A comparison of the strength of the direct dipole-dipole (blue) and the van der Waals (orange) interactions at $R_{\max }=4.6 \mu \mathrm{m}$ for different values of $n^{\prime}$ and $n=60$. (b) The exact diagonalization of the dipole-dipole interaction given in Eq. (A1). The colors indicate the projection of exact eigenstates onto the state $|1\rangle_{c}\left|r_{+}\right\rangle_{j}$. The blue dashed line denotes the eigenvalues of the perturbative Hamiltonian given in Eq. (8). 
TABLE II. The channels of the dipole-dipole interaction of $n P_{1 / 2}, n^{\prime} S_{1 / 2}$ Rydberg states.

\begin{tabular}{llll}
\hline (a) & $n P_{1 / 2}+n^{\prime} S_{1 / 2}$ & $\leftrightarrow$ & $n_{\beta} S_{1 / 2}+n_{\chi} P_{1 / 2}$ \\
\hline (b) & $n P_{1 / 2}+n^{\prime} S_{1 / 2}$ & $\leftrightarrow$ & $n_{\beta} D_{3 / 2}+n_{\chi} P_{3 / 2}$ \\
(c) & $n P_{1 / 2}+n^{\prime} S_{1 / 2}$ & $\leftrightarrow$ & $n_{\beta} D_{3 / 2}+n_{\chi} P_{1 / 2}$ \\
(d) & $n P_{1 / 2}+n^{\prime} S_{1 / 2}$ & $\leftrightarrow$ & $n_{\beta} S_{1 / 2}+n_{\chi} D_{3 / 2}$ \\
\hline \hline
\end{tabular}

smaller than the first one by choosing $n$ and $\Delta n$ properly. This makes the interaction essentially isotropic.

We note, however, that, even when the condition $\left|\widetilde{C}_{6}^{\prime}\right| \ll$ $\left|C_{6}^{\prime}\right|$ is satisfied, the second term in Eq. (A5) may cause unwanted transitions between the Rydberg states of the control atom, $|1\rangle_{c} \leftrightarrow\left|1^{\prime}\right\rangle_{c}$, which can bring the control atom out of the Hilbert space of interest. To suppress such transitions, we make them strongly off-resonant by imposing, e.g., an external magnetic field. Thus we assume that the Hilbert space of the control atom can be represented by the two qubit states $|0\rangle_{c}$ and $|1\rangle_{c}$ and we can describe the

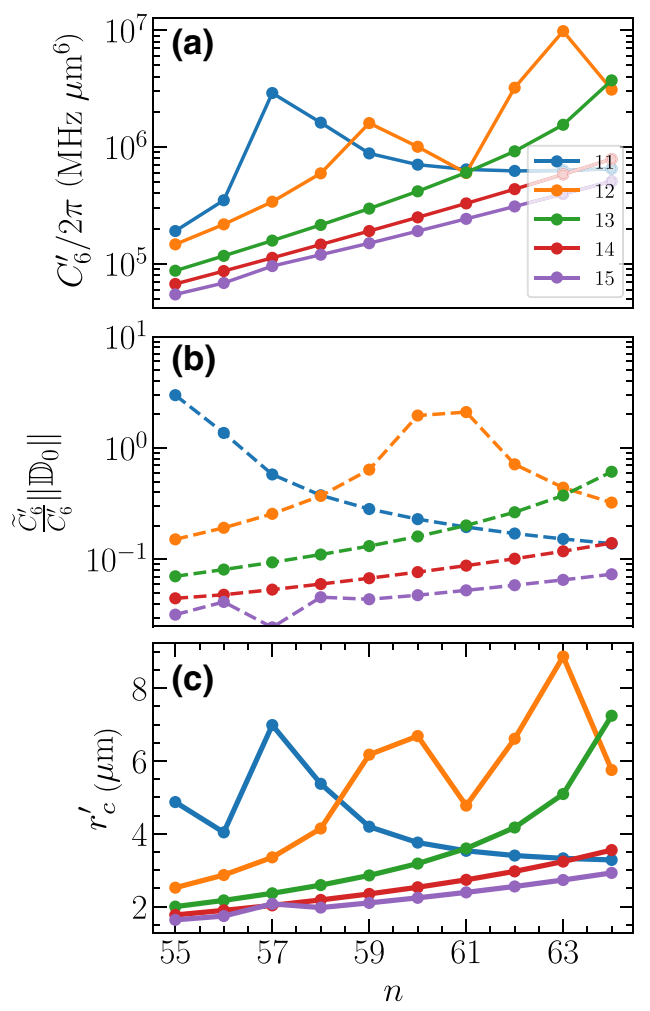

FIG. 11. The van der Waals interaction between the Rydberg states of the simulator and control atoms with the principal quantum numbers $n$ and $n^{\prime}=n+\Delta n$, respectively ( $\Delta n$ is shown in different colors as indicated in the inset). (a) The $C_{6}^{\prime}$ coefficient as a function of $n$ for different values of $\Delta n$. (b) The relative strength of two contributions to $V_{\mathrm{vdW}}^{(i, c)}$, where $\left\|\mathbb{D}_{0}\right\|=2 \sqrt{2} / 27$ is the Frobenius norm of the matrix $\mathbb{D}_{0}$. (c) The critical distance $r_{c}^{\prime}$ for the van der Waals interaction between the simulator and control atoms (see the text). interaction between the simulator atom and the $c$ qubit by the "blockade" Hamiltonian (8). The comparison with the exact diagonalization is shown in Fig. 10(b).

\section{Validity of the van der Waals Hamiltonian}

Before proceeding, we emphasize that the validity of the van der Waals interaction Hamiltonian Eq. (A2) requires the conditions $[46,84]$ to be satisfied:

$$
\begin{aligned}
\max _{\beta \chi}\left\{{ }_{i}\left\langle\left. r_{ \pm}\right|_{j}\left\langle r_{ \pm}\left|V_{d d}^{(i, j)} \delta_{\beta \chi}^{-2} Q_{\beta \chi} V_{d d}^{(i, j)}\right| r_{ \pm}\right\rangle_{i} \mid r_{ \pm}\right\rangle_{j}\right\} & \ll 1 \\
\max _{\beta \chi}\left\{{ }_{c}\left\langle\left. 1\right|_{i}\left\langle r_{ \pm}\left|V_{d d}^{(i, j)} \delta_{\beta \chi}^{-2} Q_{\beta \chi} V_{d d}^{(i, j)}\right| r_{ \pm}\right\rangle_{i} \mid 1\right\rangle_{c}\right\} & \ll 1
\end{aligned}
$$

which sets the lower bound on the interatomic distance, $r_{i j}>r_{c}$, for $i, j=1, \ldots, L$, and $r_{i, c}>r_{c}^{\prime}$, where the critical distances $r_{c}$ and $r_{c}^{\prime}$ are defined as the distances when the left-hand side in the previous condition equals unity. The calculated dependencies of $r_{c}$ and $r_{c}^{\prime}$ on the main quantum numbers are shown in the inset of Fig. 9(a) and in Fig. 11(c), respectively. For the simulator atoms, however, direct diagonalization of $V_{\mathrm{dd}}^{(i, j)}$ [Eq. (A1)] shows the presence of an avoided crossing at $r_{i j} \approx 2.4 \mu \mathrm{m}$ [see Fig. 9(b)]. Therefore, to stay away from this nonperturbative situation, we take $r_{c}=2.4 \mu \mathrm{m}$ (using $n=60$ and $n^{\prime}=71$ as principal quantum numbers) for the shortest interatomic distances in our setup.

\section{APPENDIX B: DERIVATION OF THE EFFECTIVE HAMILTONIAN}

In this appendix, we derive the effective Hamiltonian of the simulator-qubit interaction, which we show to be given by Eq. (9). We perform an adiabatic elimination of excited Rydberg states of simulator atoms $\left|r_{ \pm}\right\rangle$, following Ref. [43]. We first transform Hamiltonian $H_{s}$ [see Eqs. (4)-(6)] into a rotating frame:

$$
H_{S}=\sum_{i=1}^{L} H_{0}^{(i)}+\sum_{i, j=1}^{L} H_{\mathrm{vdW}}^{(i, j)}
$$

where

$$
\begin{aligned}
H_{0}^{(i)}= & \sum_{\alpha= \pm}\left(\Omega_{\alpha}\left|g_{\alpha}\right\rangle\left\langle g_{\alpha}\right|+\text { H.c. }\right) \\
& -\Delta_{B}\left(\left|r_{-}\right\rangle_{i}\left\langle r_{-}|+| g_{-}\right\rangle_{i}\left\langle g_{-}\right|\right) \\
& -\sum_{\alpha= \pm} \Delta_{\alpha}\left|r_{\alpha}\right\rangle_{i}\left\langle r_{\alpha}\right|,
\end{aligned}
$$

and $\quad \Delta_{B}=\omega_{+}-\omega_{-}+E_{g_{+}}-E_{g_{-}}, \quad \Delta_{ \pm}=E_{r \pm}-E_{g \pm}-$ $\omega_{ \pm}$. The explicit expression of van der Waals interaction 
Hamiltonian according to Eq. (A3) is

$$
\begin{aligned}
H_{\mathrm{vdW}}^{(i, j)}= & \sum_{\alpha, \alpha^{\prime}} W_{\alpha \alpha^{\prime}}^{(i, j)}\left|r_{\alpha}\right\rangle_{i}\left\langle r_{\alpha}|\otimes| r_{\alpha^{\prime}}\right\rangle_{j}\left\langle r_{\alpha^{\prime}}\right| \\
& +V_{+-}^{(i, j)}\left|r_{+}\right\rangle_{i}\left\langle r_{-}|\otimes| r_{-}\right\rangle_{j}\left\langle r_{+}\right|+\text {H.c. } \\
& +V_{--}^{(i, j)}\left|r_{+}\right\rangle_{i}\left\langle r_{-}|\otimes| r_{+}\right\rangle_{j}\left\langle r_{-}\right|+\text {H.c. }
\end{aligned}
$$

We now define the dressed ground $\left|\tilde{g}_{\alpha}\right\rangle_{i}$ and Rydberg $\left|\tilde{r}_{\alpha}\right\rangle_{i}$ states as the eigenstates of the Hamiltonian $H_{0}^{(i)}$,

$$
\begin{aligned}
\left|\tilde{g}_{\alpha}\right\rangle_{i} & =\frac{\sqrt{\lambda_{-}}\left|g_{\alpha}\right\rangle_{i}-\sqrt{\lambda_{+}}\left|r_{\alpha}\right\rangle_{i}}{\sqrt{\lambda_{+}+\lambda_{-}}}, \\
\left|\tilde{r}_{\alpha}\right\rangle_{i} & =\frac{\sqrt{\lambda_{+}}\left|g_{\alpha}\right\rangle_{i}+\sqrt{\lambda_{-}}\left|r_{\alpha}\right\rangle_{i}}{\sqrt{\lambda_{+}+\lambda_{-}}},
\end{aligned}
$$

where $\quad \lambda_{ \pm}=\left[\left(\Delta_{\alpha} / 2\right)^{2}+\Omega_{\alpha}^{2}\right]^{1 / 2} \pm \Delta_{\alpha} / 2 \quad$ (we assume $\Delta_{\alpha}>0$ and real Rabi frequencies $\Omega_{\alpha}$ ).

We now define the projectors onto the subspace of the dressed ground states: $\left|\tilde{g}_{\alpha}\right\rangle_{i}$

$$
P_{g}=\bigotimes_{i=1}^{L}\left(\sum_{\alpha= \pm}\left|\tilde{g}_{\alpha}\right\rangle_{i}\left\langle\tilde{g}_{\alpha}\right|\right)
$$

and $Q=\bigotimes_{i=1}^{L} \mathbb{I}_{i}-P_{g}$. Equivalently, we can write $P_{g}$ as a sum of projectors onto the eigenstates of the Hamiltonian $\sum_{i} H_{0}^{(i)}$ with the eigenenergy $E_{l}^{(0)}, P_{g}=\sum_{l} P_{g}^{(l)}$, where $P_{g}^{(l)}$ is the projector onto the subspace formed by the eigenstate with the eigenenergy $E_{l}^{(0)}$. Due to the particular form of the blockade interaction given in Eq. (A5), we can perform the adiabatic elimination of excited states simultaneously for the two states of the qubit. We now derive the effective Hamiltonian of the simulator atoms using projectors and write an effective Hamiltonian in the $P_{g}$ subspace up to two lowest orders in $V$ as [85]

$$
\begin{aligned}
H_{\mathrm{eff}}= & P_{g} H_{s} P_{g}+\frac{1}{2} \\
& \times\left\{P_{g} H_{s} Q \sum_{l}\left(E_{l}^{(0)}-Q H_{s} Q\right)^{-1} Q H_{s} P_{g}^{(l)}+\text { H.c. }\right\} .
\end{aligned}
$$

After evaluating this expression analytically up to the fourth order in $\xi_{\alpha} \equiv \Omega_{\alpha} / \Delta_{\alpha}$, we obtain [43] the effective Hamiltonian in general $X Y Z$ form:

$$
\begin{aligned}
H_{\mathrm{eff}}\left(\Delta_{\alpha}\right)= & \sum_{i<j=1}^{L} \sum_{\eta=x, y, z} J_{i j}^{(\eta)}\left(\Delta_{\alpha}\right) \sigma_{i}^{\eta} \sigma_{j}^{\eta} \\
& +\sum_{i=1}^{L}\left[h^{z}\left(\Delta_{\alpha}\right)+\frac{1}{2} \Delta_{B}\right] \sigma_{i}^{z} .
\end{aligned}
$$

We now show that the model can be reduced to the Heisenberg $X X Z$ model assumed in the main text and provide the corresponding interaction coefficients $J_{i j}^{(\eta)}, h^{z}$. The expressions for the Hamiltonians $H_{\text {spin }}$ and $H_{\text {spin }}^{\prime}$ in Eq. (9) are as follows:

$$
\begin{gathered}
H_{\text {spin }}=H_{\text {eff }}\left(\Delta_{\alpha}\right), \\
H_{\text {spin }}^{\prime}=H_{\text {eff }}\left(\Delta_{\alpha}-C_{6}^{\prime} / R^{6}\right) .
\end{gathered}
$$

As a result of the adiabatic elimination procedure, the qubit logical states $|0\rangle_{c}$ and $|1\rangle_{c}$, respectively, acquire additional spin-independent shifts $\sum \beta_{i j}$ and $\sum \beta_{i j}^{\prime}$ (see the following). Thus in the rotating frame of the control laser frequency, the Hamiltonian takes on the form Eq. (9) with an effective detuning $\delta=\sum_{i j}\left(\beta_{i j}^{\prime}-\beta_{i j}^{\prime}\right)+E_{\mathrm{cr}}-E_{\mathrm{cg}}$.

We now discuss the effective Hamiltonians (B7) and (B8). In the case of a perfect blockade, $C_{6}^{\prime} /\left(\Delta_{\alpha} R^{6}\right) \rightarrow \infty$, one has $J_{i j}^{(\eta)}\left(\Delta_{\alpha}-C_{6}^{\prime} / R^{6}\right) \rightarrow 0$ and $h_{i}^{z}\left(\Delta_{\alpha}-C_{6}^{\prime} / R^{6}\right) \rightarrow$ 0 and, therefore, $H_{\text {spin }}^{\prime}$ reduces to a simple form $H_{\text {spin }}^{\prime}=$ $(1 / 2) \Delta_{B} \sum_{i=1}^{L} \sigma_{i}^{z}$. With this, we are able to identify two regimes in which the Hamiltonian given in Eq. (9) gives rise to the desired QND Hamiltonian, i.e., $\left[H_{\text {spin }}, H_{\text {spin }}^{\prime}\right]=$ 0 . The first one corresponds to $\Delta_{B}=0$. In this paper, we focus on the second regime of a "strong magnetic field" when $\Delta_{B} \rightarrow \infty$ (more precisely, $\left|\Delta_{B}\right| \gg\left|J_{i j}^{(\eta)}\right|,\left|h_{i}^{z}\right|$ ), when the spin model effectively reduces to the Heisenberg $X X Z$ chain. The explicit expressions for the couplings are then given by

$$
\begin{aligned}
& h^{z}=0, \\
& J_{i j}^{(x, y)}=-2 \Delta^{2} \xi^{4} \frac{V_{+-}^{(i, j)}}{\left(V_{+-}^{(i, j)}-W_{+-}^{(i, j)}-2 \Delta\right)\left(2 \Delta+V_{+-}^{(i, j)}+W_{+-}^{(i, j)}\right)}, \\
& J_{i j}^{(z)}=-2 \Delta^{2} \xi^{4} \frac{V_{+-}^{2}-\left(2 \Delta+W_{+-}^{(i, j)}\right)\left(W_{+-}^{(i, j)}-W_{++}^{(i, j)}\right)}{\left(2 \Delta+W_{+-}^{(i, j)}\right)\left(V_{+-}^{(i, j)}-W_{+-}^{(i, j)}-2 \Delta\right)\left(2 \Delta+V_{+-}^{(i, j)}+W_{+-}^{(i, j)}\right)},
\end{aligned}
$$




$$
\beta_{i j}=-2 \Delta \xi^{2}+2 \Delta \xi^{4} \frac{V_{+-}^{2}\left(3 \Delta+2 W_{++}^{(i, j)}\right)-\left(2 \Delta+W_{+-}^{(i, j)}\right)\left[4 \Delta^{2}+3 \Delta\left(W_{+-}^{(i, j)}+W_{++}^{(i, j)}\right)+2 W_{+-}^{(i, j)} W_{++}^{(i, j)}\right]}{\left(2 \Delta+W_{++}^{(i, j)}\right)\left(V_{+-}^{(i, j)}-2 \Delta-W_{+-}^{(i, j)}\right)\left(2 \Delta+V_{+-}^{(i, j)}+W_{+-}^{(i, j)}\right)}
$$

where $V$ and $W$ refer to the van der Waals interaction derived in Eq. (1) and we assume equal Rabi frequencies and detunings, such that $\xi_{\alpha}=\xi$. If the energy difference between the subspaces is much larger than the coupling $\left|J_{i j}^{(\eta)}\right| \ll\left|\Delta_{B}\right|$, this term can be neglected, such that the resulting low-energy dynamics (with typical energies of approximately $J$ ) takes place in a sector with a fixed $S_{z}=$ $\sum_{i} \sigma_{i}^{z}$.

To conclude, we note that in Eq. (B6), one can also generate aposition-dependent magnetic field, $h^{z} \rightarrow h_{i}^{z}=$ $h^{z}+\delta h_{i}^{z}$. This can be achieved by using position dependent Rabi frequencies, $\Omega_{\alpha}^{(i)}=\Omega_{\alpha}+\delta \Omega_{\alpha}^{(i)}$, which results in $\delta h_{i}^{z} \approx \sum_{\alpha} \alpha \xi_{\alpha} \delta \Omega_{\alpha}^{(i)}$. After choosing $\delta \Omega_{\alpha}^{(i)} \sim(J / \xi) w_{i}$, where $w_{i} \in[-1,1]$ is a uniformly distributed random number, we obtain $\delta h_{i}^{z} \sim J$.

\section{APPENDIX C: IMPLEMENTATION OF COMPLEX FLIP-FLOP PHASES}

We now provide a way to engineer complex flip-flop coefficients in our spin models. As a starting point, let us consider the dressing lasers with the Laguerre-Gaussian spatial mode profile corresponding to the angular momentum $l_{ \pm}$(note that it is sufficient to have only one nonzero $l_{\alpha}$ ). In this case, the dressing term in the Hamiltonian given in Eq. (4) becomes

$$
\sum_{k=1}^{L} \sum_{\alpha= \pm}\left(\Omega_{\alpha}^{\mathrm{LG}} e^{i \phi_{k, \alpha}}\left|g_{\alpha}\right\rangle_{k}\left\langle r_{\alpha}\right| e^{i \omega_{\alpha} t}+\text { H.c. }\right)
$$

where $\phi_{k, \pm} \equiv 2 \pi k l_{ \pm} / L$. After performing the adiabatic elimination procedure as in Appendix B, we obtain the effective Hamiltonian of the form following form (here, we omit the $\Delta_{B}$ term):

$$
\begin{aligned}
H_{\text {eff }}\left(\tilde{\Delta}_{\alpha}\right)= & \sum_{i<j=1}^{L}\left\{2 J_{k j}^{(x, y)}\left(e^{i \sum_{\alpha} \alpha\left(\phi_{k, \alpha}-\phi_{j, \alpha}\right)} \sigma_{k}^{+} \sigma_{j}^{-}+\text {H.c. }\right)\right\} \\
& +\sum_{i<j=1}^{L}\left\{J_{k j}^{(z)} \sigma_{k}^{z} \sigma_{j}^{z}\right\}+\sum_{i=1}^{L} h_{i}^{z} \sigma_{i}^{z},
\end{aligned}
$$

with the flip-flop amplitudes acquiring nonzero phases $\phi_{k j}=i \sum \alpha\left(\phi_{k, \alpha}-\phi_{j, \alpha}\right)$. We note, however, that the sum of the flip-flop phases in Eq. (C2) across the system is always equal to an integer number of $2 \pi$, having, therefore, no effect on the spectral statistics. However, the physically relevant phase of the flip-flop amplitudes can be generated using stroboscopic engineering. Let us consider the case when, during the stroboscopic period $T=2 \pi / \omega_{s}$, we use the plane-wave dressing lasers with the Rabi frequencies $\Omega_{\alpha}$ for the time $0 \leq t_{1} \leq T$ and the Laguerre-Gaussian dressing lasers with the Rabi frequencies $\Omega_{\alpha}^{\mathrm{LG}}$ for the time $t_{2}=T-t_{1}$. Then, for the stroboscopic frequency satisfying the condition $h, J \ll \omega_{s} \ll \Delta_{ \pm}$, the effective Rabi frequencies at the atomic positions are

$$
\Omega_{\alpha, k}^{\mathrm{eff}}=\frac{1}{T}\left(t_{1} \Omega_{\alpha}+t_{2} \Omega_{\alpha}^{\mathrm{LG}} e^{i \phi_{k, \alpha}}\right)=\left|\Omega_{\alpha}^{\mathrm{eff}}\right| e^{i \phi_{k, \alpha}^{\mathrm{eff}}},
$$

where

$$
\begin{aligned}
\left|\Omega_{\alpha}^{\mathrm{eff}}\right|= & \frac{1}{T^{2}}\left[\left(t_{1} \Omega_{\alpha}+t_{2} \Omega_{\alpha}^{\mathrm{LG}} \cos \phi_{k, \alpha}\right)^{2}\right. \\
& \left.+\left(t_{2} \Omega_{\alpha}^{\mathrm{LG}} \sin \phi_{k, \alpha}\right)^{2}\right]^{1 / 2}
\end{aligned}
$$

and

$$
\tan \phi_{k, \alpha}^{\mathrm{eff}}=\frac{\sin \phi_{k, \alpha}}{\cos \phi_{k, \alpha}+t_{1} / t_{2}} .
$$

We see that by varying the ratio $t_{1} / t_{2}$, one can generate values $\phi_{k, \alpha}^{\text {eff }} \in\left[0, \phi_{k, \alpha}\right]$ for the effective phases. With the effective Rabi frequencies (C3), the resulting phases of the flip-flop terms are no longer multiples of $2 \pi$ and the corresponding Hamiltonian (C2) now belongs to the unitary ensemble.

\section{APPENDIX D: EXPERIMENTAL CONSIDERATIONS FOR SFF}

In this appendix, we discuss in more detail the experimental challenges of the SFF protocol, which limit the achievable system sizes. We also present estimations of the coherence times and the available system sizes for our Rydberg-tweezer implementation.

Time scales. Propagation up to the Heisenberg time $\tau_{H} \sim 2^{L} / J L$ is limited by the finite coherence time of the quantum simulator. First, the preparation time (with $M$ measurements) is $t_{\text {prep }} \approx t_{0} 2^{M} \leq \pi(J L)^{-1} 2^{M-1}$ (see Sec. III B 1). Therefore, the lower bound on the coherence time is $t_{\text {coh }}>t_{\text {prep }} \approx(J L)^{-1} 2^{M-1}$. Observation of the behavior of $K(\tau)$ at times $\tau \sim \tau_{\mathrm{H}}$, requires coherence times $t_{\text {coh }}>\tau_{\mathrm{H}} \sim(J L)^{-1} 2^{L}$. In the case of a dominant individual single-spin decoherence with the dephasing rate $\gamma_{\mathrm{d}}$, the corresponding condition reads $J / \gamma_{\mathrm{d}}>2^{L}$. This limits observation of $K(\tau)$ at times $\tau \sim \tau_{H}$ to moderate 
system sizes (see estimates for the Rydberg-tweezer array in the following). We note, however, that even when the Heisenberg time $\tau_{H}$ is not accessible, the transition to chaotic dynamics at the Thouless time $\tau_{\mathrm{Th}}$ and the distinct behaviors of the SFF for quantum chaotic $[K(\tau) \sim \tau]$ and integrable systems $[K(\tau) \sim$ const $]$ take place at much shorter times in comparison with $t_{\text {coh. }}$. In general, systems with smaller $L$ are characterized by shorter characteristic times and require fewer experimental runs to resolve the key features (see the discussion in the following). On the other hand, finite-size effects tend to wash out the characteristic features of $K(\tau)$, signaling the chaotic behavior.

Signal magnitude. The scaling of typical SFF values can be estimated as $K_{\infty} \sim N_{\Delta E}^{-1}$ for the late-time plateau value and as $K\left(\tau \approx \tau_{\mathrm{Th}}\right) \sim N_{\Delta E}^{-3 / 2}$ [86] for the SFF minimum preceding the Thouless time $\tau_{\mathrm{Th}}$, here $N_{\Delta E} \gg 1$ is the number of eigenstates in the initial MC ensemble $\rho_{\mathrm{mc}}$. Preparation of the MC ensemble (see Sec. III B 1) using $M$ filtering steps ( $c$ qubits) produces $\rho_{\mathrm{mc}}$ with $N_{\Delta E} \sim 2^{L-M}$ eigenstates. Since the protocol has a success probability $p_{\mathrm{mc}} \sim 2^{-M}$ and the QND measurement scheme can exploit the prepared state several times $\left(N_{\text {reuse }}\right)$, one can perform $N$ measurements per data point in $N_{\text {run }}^{(1)} \simeq N /\left(p_{\text {mc }} N_{\text {reuse }}\right)$ experimental runs per one disorder realization. On the other hand, the threshold signal level that can be distinguished from the shot noise after averaging over $N_{\mathrm{d}}$ realizations of disorder in the spin Hamiltonian and $N$ measurements per one disorder is given by $K_{*} \sim 1 /\left(N \sqrt{N_{\mathrm{d}}}\right)$ (Appendix F). We find the necessary number of measurements using the condition $K_{*} \sim K_{\infty}$. Thus, the number of experimental runs $N_{\text {run }}=N_{\mathrm{d}} N_{\text {run }}^{(1)}$ per data point necessary to resolve the features of interest in $K(\tau)$, is given by $N_{\text {run }}>2^{L} \sqrt{N_{\mathrm{d}}} / N_{\text {reuse }}$.

The number of filtering steps $M$ does not affect $N_{\text {run }}$ for the probabilistic preparation scheme; therefore, it is enough to use $M \sim 3$ to eliminate the contribution of spectral edges in the SFF. However, it might be possible to use a semideterministic preparation scheme, e.g., by populating excited energy eigenstates in some energy interval by driving or quenching the system [40] followed by verification via $M$ filtering steps with a high probability (approximately 1) of success. In this case, the necessary number of experimental runs per data point can be improved to $N_{\text {run }}>2^{L-M}$.

Rydberg-tweezer implementation:- We conclude with a discussion of imperfections for the Rydberg-tweezer implementation; in particular, decoherence rates and the effectiveness of the Rydberg blockade. We also elaborate on geometrical limitations on interatomic distances that ensure the validity of our Rydberg dressing and put a constraint on the maximal size of the system.

We start with the discussion of the decoherence effects that limit the duration of the experiment. They originate from the finite lifetime of the Rydberg atomic states and from the error rate in the gate operation caused by an imperfect Rydberg-blockade mechanism [the term with $H_{\text {spin }}^{\prime}$ in (9)]. The former is characterized by two dimensionless parameters, $\kappa_{1} \equiv \gamma_{d}^{\prime} /\left|H_{\text {spin }}\right| \sim \gamma_{d}^{\prime} /(J L)$ and $\kappa_{2} \equiv$ $\xi_{ \pm}^{2} \gamma_{d} L /\left|H_{\text {spin }}\right| \sim \xi_{ \pm}^{2} \gamma_{d} / J$, for the control atom and the spin system, respectively. Here, $\gamma_{d}^{\prime}$ and $\gamma_{d}$ are the spontaneous emission rates for the corresponding Rydberg states, and for the system atoms we take into account the collective enhancement (approximately $L$ ) of the spontaneous emission rate due to highly entangled nature of the many-body excited states. The factor $\xi_{ \pm}^{2}$ in $\kappa_{2}$ represents the admixture of the Rydberg state as a result of the dressing. In a similar way, the error rate in the gate operation can be quantified by the parameter $\kappa_{3} \equiv\left|H_{\text {spin }}^{\prime}\right| /\left|H_{\text {spin }}\right|$, which can be estimated [see Eqs. (B10) and (B11)] as $\kappa_{3} \sim\left(R / R_{b}\right)^{24}$ for $R<R_{\mathrm{b}}$, where $R$ is the distance between the control atom and the system atoms and $R_{b} \equiv \sqrt[6]{\left|C_{6}^{\prime} / \Delta_{ \pm}\right|}$is the Rydbergblockade radius, in which $C_{6}^{\prime}$ is the interaction constant [see Eq. (8)]. We note here the very high power in the previous estimate such that $\kappa_{3}$ decreases very rapidly for $R<$ $R_{b}$, giving, e.g., $\kappa_{3} \sim 10^{-3}$ for $R / R_{b}=0.8$. The largest of these parameters $\kappa=\max \left\{\kappa_{i}\right\}$ sets the upper bound for the time during which the evolution is coherent and follows the ideal QND Hamiltonian (1), $t_{\mathrm{coh}} \sim(J L \kappa)^{-1}$.

As an example, let us consider the Rydberg states with $n^{\prime}=71$ (control atom) $n=60$ (system atom) and the following parameters of the dressing scheme: $\Delta_{ \pm}=-9 \mathrm{MHz}$ and $\xi_{ \pm}=0.2$. The decoherence rates for the atoms are $\gamma_{d} \approx 2 \pi \times 318 \mathrm{~Hz}$ and $\gamma_{d}^{\prime} \approx 2 \pi \times 406 \mathrm{~Hz}$. The parameters characterizing the spontaneous emission are then $\kappa_{1} \approx 4.4 \cdot 10^{-4}, \kappa_{2}=1.6 \cdot 10^{-4}$ for $L \sim 10$ atoms and the Rydberg-blockade radius is $R_{b} \approx 6.5 \mu \mathrm{m}$ (see Fig. 11). For $\kappa_{3}$ to be of the same order or smaller, one should have $R \sim R_{\max }=0.75 R_{b} \approx 5 \mu \mathrm{m}$ as an upper bound on the distance between the system and control atoms. In our system, $\kappa_{3}$ is the largest decoherence parameter and it limits the coherence times to $t_{\mathrm{coh}} \sim 10^{2} \mathrm{~J}^{-1}$.

The constraint on the distance between the system atoms is related to the validity of our dressing scheme, i.e., the validity of the perturbative approach: it has to be larger than some minimal value $R_{i j}>r_{c}=2.4 \mu \mathrm{m}$ (see Appendix A 2). Within our ring setup [see Fig. 1(b)], in which the radius is given by $R_{\max }$ and the separation between system atoms is limited by $r_{c}$, simple geometrical considerations give the maximal number of system atoms, which is, with both constraints, $L_{\max } \simeq \pi / \arcsin \left[r_{c} /\left(2 R_{\max }\right)\right]=12$, the number used in our numerical simulations.

\section{APPENDIX E: PREPARATION OF A MICROCANONICAL ENSEMBLE}

In this appendix, we present a detailed description of the preparation of microcanonical ensembles via 
low-resolution PEA, as discussed in Sec. III B 1. As explained in the main text, the preparation procedure involves $M$ rounds of the QND interaction entangling the spin system and the $c$ qubit according to $\mathcal{U}\left(t_{m}, \delta\right)=$ $\exp \left\{-i\left[\left(H_{\text {spin }}-\delta\right) \otimes|0\rangle\langle 0|\right] t_{m}\right\}$ followed by qubit measurements.

After the entanglement, the measurement of the $c$ qubit with a measurement outcome $v_{m}=\{ \pm\}$ collapses a state $\rho$ of the spin system into a conditional (unnormalized) state

$$
\rho \rightarrow \mathcal{M}\left(v_{m}, t_{m}, \delta\right) \rho \mathcal{M}^{\dagger}\left(v_{m}, t_{m}, \delta\right),
$$

where the measurement operators $\mathcal{M}( \pm, t, \delta)=$ $\langle \pm|\mathcal{U}(t, \delta)|+\rangle$ act in the system Hilbert space and have a diagonal representation in the energy eigenbasis

$$
\begin{aligned}
& \mathcal{M}(+, t, \delta)=\sum_{\ell} e^{-i\left(E_{\ell}-\delta\right) t / 2} \cos \left[\left(E_{\ell}-\delta\right) t / 2\right]|\ell\rangle\langle\ell|, \\
& \mathcal{M}(-, t, \delta)=i \sum_{\ell} e^{-i\left(E_{\ell}-\delta\right) t / 2} \sin \left[\left(E_{\ell}-\delta\right) t / 2\right]|\ell\rangle\langle\ell| .
\end{aligned}
$$

The full sequence of $c$-qubit measurements with outcomes $\vec{v} \equiv\left\{v_{m}\right\}$ defines the resulting unnormalized state of the spin system:

$$
\tilde{\rho}_{\text {out }}=\mathbb{M}(\vec{v}, \delta) \rho_{\text {in }} \mathbb{M}^{\dagger}(\vec{v}, \delta),
$$

where $\mathbb{M}(\vec{v}, \delta)=\prod_{m=0}^{M-1} \mathcal{M}\left(v_{m}, t_{m}, \delta\right)$. As a result, the unnormalized probability $p_{\ell}$ for the eigenstates $|\ell\rangle$ with the eigenenergy $E_{\ell}$ to appear in $\rho_{\text {out }}$ is

$$
\begin{aligned}
p_{\ell}=\left\langle\ell\left|\tilde{\rho}_{\text {out }}\right| \ell\right\rangle & =P\left(\vec{v}, E_{\ell}-\delta\right)\left\langle\ell\left|\rho_{\text {in }}\right| \ell\right\rangle, \\
P(\vec{v}, x) & =\prod_{m=0}^{M-1} P_{m}\left(v_{m}, x\right)
\end{aligned}
$$

where $P_{m}( \pm, x)=\left\{1 \pm \cos \left(x t_{m}\right)\right\} / 2$.

If we now select a run with all readouts " + ," $v_{m}=$ $\{+\}, m=0, \ldots, M-1$, we obtain the output state with the narrow energy distribution

$$
\begin{gathered}
p_{\ell}=P\left(+_{0}, \ldots+_{M-1}, E_{\ell}-\delta\right)\left\langle\ell\left|\rho_{\text {in }}\right| \ell\right\rangle, \\
P\left(+_{0}, \ldots+_{M-1}, x\right)=\left\{\frac{\sin \left(2^{M} t_{0} x\right)}{2^{M} \sin \left(t_{0} x\right)}\right\}^{2} .
\end{gathered}
$$

The success probability of the protocol is $p_{\mathrm{mc}} \equiv \sum_{\ell} p_{\ell}$. Expressions (E3) and (E4) are used in Sec. III B 1.

\section{APPENDIX F: SHOT NOISE IN SFF MEASUREMENTS}

Here, we determine the shot noise in the SFF measurement. As described in Secs. III and IV, the measurement of the SFF involves the estimation of the expectation values $\left\langle\sigma^{x, y}\right\rangle$ for the $c$ qubit by averaging results of $N$ measurements. The statistical properties of such averaging can be described by introducing $N$ copies of the $c$ qubit and the spin system and considering the fluctuations of the collective spin $S_{x, y}=N^{-1} \sum_{k=1}^{N} \sigma_{k}^{x, y}$ of the $N c$ qubits. In particular, we consider each copy of the $c$ qubit and spin system to be initialized in the states $|+\rangle=\frac{1}{\sqrt{2}}(|0\rangle+|1\rangle)$ and $\rho_{\text {in }}$, respectively, and entangled via a controlled unitary $\mathcal{U}(\tau)=U(\tau) \otimes|0\rangle\langle 0|+\mathbb{I} \otimes| 1\rangle\langle 1|$. In the case of Hamiltonian dynamics, we have $\mathcal{U}(\tau)=e^{-i \mathcal{H}_{\mathrm{QND}} \tau}$ and, consequently, $U(\tau)=e^{-i H_{\text {spin }} \tau}$. Since we are interested in the noise due to a finite number of measurements, in what follows, we consider a specific and fixed realization of disorder in the Hamiltonian $H_{\text {spin }}$.

After the QND interaction, the state of the $N$ copies of the system reads

$$
\rho(\tau)=\left[\mathcal{U}(\tau) \rho_{\text {in }} \otimes|+\rangle\langle+| \mathcal{U}(\tau)^{\dagger}\right]^{\otimes N} .
$$

The consecutive measurements of the $c$ qubits yield an averaged outcome that is given by an eigenvalue $m_{x, y}$ of the observable $S_{x, y}$. The statistical distribution of the outcomes $m_{x, y}$ is characterized by the corresponding moments of the collective spin observables with respect to the state $\rho(\tau)$.

The mean values read

$$
\begin{aligned}
\left\langle S_{x}(\tau)\right\rangle & =\frac{1}{N} \sum_{k=1}^{N} \operatorname{Tr}\left\{\rho(\tau)\left(\mathbb{I} \otimes|0\rangle_{k}\langle 1|+\mathbb{I} \otimes| 1\rangle_{k}\langle 0|\right)\right\} \\
& =\frac{1}{2} \operatorname{Tr}\left\{\rho_{\text {in }}\left[U(\tau)+U(\tau)^{\dagger}\right]\right\}=\operatorname{Re}\langle U(\tau)\rangle, \\
\left\langle S_{y}(\tau)\right\rangle & =\operatorname{Im}\langle U(\tau)\rangle
\end{aligned}
$$

where we express the operators $\sigma_{k}^{x, y}$ explicitly in the basis $|0(1)\rangle_{k}$ of the $k$ th $c$ qubit. Thus, one can use the first moments to evaluate the average of the unitary operator $\langle U(\tau)\rangle=\left\langle S_{x}(\tau)\right\rangle+i\left\langle S_{y}(\tau)\right\rangle \approx m_{x}+i m_{y}$.

The second and fourth moments of $S_{x}$ read

$$
\begin{aligned}
\left\langle S_{x}^{2}(\tau)\right\rangle= & \frac{1}{N^{2}}\left\{N(N-1)[\operatorname{Re}\langle U(\tau)\rangle]^{2}+N\right\}, \\
\left\langle S_{x}^{4}(\tau)\right\rangle= & \frac{1}{N^{4}}\left\{N(N-1)(N-2)(N-3)[\operatorname{Re}\langle U(\tau)\rangle]^{4}\right. \\
& +2 N(N-1)(3 N-4)[\operatorname{Re}\langle U(\tau)\rangle]^{2} \\
& +N(3 N-2)\} .
\end{aligned}
$$

This result and similar expressions for $S_{y}$ allow us to express the SFF and its fluctuations (for a single disorder 
realization) as

$$
\begin{aligned}
K(\tau) & \equiv[\operatorname{Re}\langle U(\tau)\rangle]^{2}+[\operatorname{Im}\langle U(\tau)\rangle]^{2} \\
& =\frac{N}{N-1}\left(\left\langle S_{x}^{2}(\tau)\right\rangle+\left\langle S_{y}^{2}(\tau)\right\rangle-\frac{2}{N}\right) \\
& \approx m_{x}^{2}+m_{y}^{2}-\frac{2}{N} \pm \mathcal{O}\left(\frac{1}{N}\right) .
\end{aligned}
$$

More precisely, the variance of the SFF estimation for $N \gg 1$ reads

$$
\begin{aligned}
\operatorname{var}[K(\tau)] & =\left\langle\left[S_{x}(\tau)^{2}+S_{y}(\tau)^{2}\right]^{2}\right\rangle-\left[\left\langle S_{x}^{2}(\tau)\right\rangle+\left\langle S_{y}^{2}(\tau)\right\rangle\right]^{2} \\
& \approx \frac{4}{N} K(\tau)+\frac{4}{N^{2}}
\end{aligned}
$$

The SNR is thus given by $\mathrm{SNR} \equiv K(\tau) / \sqrt{\operatorname{var}[K(\tau)]}$. The SNR grows linearly SNR $\sim K(\tau) N / 2$ with $N$ up to $N \sim$ $2(1+\sqrt{2}) / K(\tau)$, where the SNR becomes 1 . For a given number $N$ of measurements and a fixed disorder, values of the SFF above the threshold of $K_{*}^{(1)} \equiv 2(1+\sqrt{2}) / N$ can thus be determined with an SNR that is larger than 1 . The threshold value averaged over $N_{\mathrm{d}}$ realization of disorder $K_{*}=K_{*}^{(1)} / \sqrt{N_{\mathrm{d}}}$ is presented in Sec. III B 3 and shown as horizontal lines in Figs. 3 and 6. A further increase of the number of measurements results in a slower growth with $\mathrm{SNR} \sim \sqrt{K(\tau) N} / 2$.

\section{APPENDIX G: RECYCLING OF THE MICROCANONICAL STATE IN THE SFF MEASUREMENT}

In the appendix, we show that the SFF can be measured sequentially at different times $\tau_{i}$ using a single initial state $\rho_{\mathrm{mc}}$. Since the state $\rho_{\mathrm{mc}}$ is diagonal in the energy basis, it commutes with the QND Hamiltonian (1). Therefore, the state is not perturbed after averaging over measurement results for a certain time $\tau$ :

$$
\begin{aligned}
\rho_{\text {out }}= & \mathcal{M}(+, \tau, \delta) \rho_{\mathrm{mc}} \mathcal{M}^{\dagger}(+, \tau, \delta) \\
& +\mathcal{M}(-, \tau, \delta) \rho_{\mathrm{mc}} \mathcal{M}^{\dagger}(-, \tau, \delta) \\
= & \rho_{\mathrm{mc}}
\end{aligned}
$$

Here, $\mathcal{M}( \pm, \tau, \delta)$ are the measurement operators defined in Eqs. (E1) and (E2).

Consequently, one can recycle the prepared microcanonical state as long as the decoherence in the spin system is negligible $\sum_{i} \tau_{i} \ll t_{\text {coh }}$. Furthermore, this applies to any initial state, as only the diagonal part of its density matrix contributes to the SFF. This result is used in Secs. III B 2 and III B 4.

\section{APPENDIX H: MEASUREMENT OF THE HEISENBERG TIME}

In this appendix, we show how the Heisenberg time $\tau_{H}$ and the late-time plateau of the SFF $K_{\infty}$ can be obtained from the probability $p_{\mathrm{mc}}$ with which the preparation of a microcanonical ensemble as described in Sec. III B 1 succeeds. Since the RMT form of the SFF is fully determined by these two parameters, the method we describe in the following can be used to validate the unbiased measurement of the SFF according to Sec. III in the regime in which RMT is applicable.

The preparation scheme assumes that the spin system is initialized in the infinite-temperature state $\rho_{\infty}=$ $\mathcal{D}^{-1} \sum_{\ell=1}^{\mathcal{D}}|\ell\rangle\langle\ell|$, where $\mathcal{D}$ is the Hilbert-space dimension and $|\ell\rangle$ are the eigenstates of the spin Hamiltonian. A microcanonical ensemble can then be prepared through $M$ successive projections of the $c$ qubit to the state $|+\rangle$, which occur each time after the $c$ qubit has been entangled with the spin system. The success probability for this procedure reads

$$
\begin{aligned}
p_{\mathrm{mc}} & =\frac{1}{\mathcal{D}} \sum_{\ell} P_{+M}\left(E_{\ell}-\delta\right) \\
& \approx \frac{1}{\mathcal{D}} \frac{1}{t_{0} \delta_{E}} \int_{-1}^{1} P_{+M}(x) d x \equiv \frac{1}{\mathcal{D}} \frac{1}{t_{0} \delta_{E}} \mathcal{I}_{M},
\end{aligned}
$$

where the filter function $P_{+M}(x)$ is given by the Eq. (14) and $\delta_{E}$ is the mean level spacing. Here, we assume that the filter function is narrow enough such that smooth changes in the density of states can be neglected. For $M \gg 1$, the integral $\mathcal{I}_{M}$ converges to $\pi 2^{-M}$. Therefore, the mean level spacing can be found as

$$
\delta_{E} \approx \frac{\mathcal{I}_{M}}{\mathcal{D} p_{\mathrm{mc}}} \frac{1}{t_{0}} \approx \frac{\pi 2^{-M}}{\mathcal{D} p_{\mathrm{mc}}} \frac{1}{t_{0}}
$$

and the corresponding Heisenberg time reads

$$
\tau_{H} \equiv \frac{2 \pi}{\delta_{E}} \approx 2^{M+1} t_{0} \mathcal{D} p_{\mathrm{mc}}
$$

The late-time plateau of the SFF can be estimated in a similar way. It is given by

$$
\begin{aligned}
K_{\infty} & =\sum_{\ell} f\left(E_{\ell}\right)^{2}=\frac{1}{Z^{2}} \sum_{\ell} P_{+M}\left(E_{\ell}-\delta\right)^{2} \\
& \approx \frac{1}{Z^{2}} \frac{1}{t_{0} \delta_{E}} \int_{-1}^{1} P_{+M}(x)^{2} d x \equiv \frac{\mathcal{S}_{M}}{Z^{2}} \frac{1}{t_{0} \delta_{E}}
\end{aligned}
$$

where $Z \equiv \sum_{\ell} P_{+M}\left(E_{\ell}-\delta\right) \approx \mathcal{I}_{M} /\left(t_{0} \delta_{E}\right)$. Taking into account that the integral $\mathcal{S}_{M}$ converges to $\pi 2^{1-M} / 3$ for 
$M \gg 1$ and using the expression for $p_{\mathrm{mc}}$ [Eq. (H1)], we obtain

$$
K_{\infty} \approx \frac{\mathcal{S}_{M}}{\mathcal{I}_{M}^{2}} t_{0} \delta_{E} \approx \frac{\mathcal{S}_{M}}{\mathcal{I}_{M}} \frac{1}{\mathcal{D} p_{\mathrm{mc}}} \approx \frac{2}{3} \frac{1}{\mathcal{D} p_{\mathrm{mc}}}
$$

Equations (H2) and (H3) are used in Sec. III B 1 to uniquely fix the RMT prediction using experimental data.

[1] H. Labuhn, D. Barredo, S. Ravets, S. de Léséleuc, T. Macrì, Th. Lahaye, and A. Browaeys, Tunable two-dimensional arrays of single Rydberg atoms for realizing quantum ising models, Nature 534, 667 (2016).

[2] M. A. Norcia, A. W. Young, and A. M. Kaufman, Microscopic Control and Detection of Ultracold Strontium in Optical-Tweezer Arrays, Phys. Rev. X 8, 041054 (2018).

[3] A. Cooper, J. P. Covey, I. S. Madjarov, S. G. Porsev, M. S. Safronova, and M. Endres, Alkaline-Earth Atoms in Optical Tweezers, Phys. Rev. X 8, 041055 (2018).

[4] D. Barredo, V. Lienhard, S. de Léséleuc, Th. Lahaye, and A. Browaeys, Synthetic three-dimensional atomic structures assembled atom by atom, Nature 561, 79 (2018).

[5] E. Guardado-Sanchez, P. T. Brown, D. Mitra, T. Devakul, D. A. Huse, P. Schauß, and W. S. Bakr, Probing the Quench Dynamics of Antiferromagnetic Correlations in a 2D Quantum Ising Spin System, Phys. Rev. X 8, 021069 (2018).

[6] T. L. Nguyen, J. M. Raimond, C. Sayrin, R. Cortiñas, T. Cantat-Moltrecht, F. Assemat, I. Dotsenko, S. Gleyzes, S. Haroche, G. Roux, Th. Jolicoeur, and M. Brune, Towards Quantum Simulation with Circular Rydberg Atoms, Phys. Rev. X 8, 011032 (2018).

[7] A. Piñeiro Orioli, A. Signoles, H. Wildhagen, G. Günter, J. Berges, S. Whitlock, and M. Weidemüller, Relaxation of an Isolated Dipolar-Interacting Rydberg Quantum Spin System, Phys. Rev. Lett. 120, 063601 (2018).

[8] A. Omran, H. Levine, A. Keesling, G. Semeghini, T. T. Wang, S. Ebadi, H. Bernien, A. S. Zibrov, H. Pichler, S. Choi, J. Cui, M. Rossignolo, P. Rembold, S. Montangero, T. Calarco, M. Endres, M. Greiner, V. Vuletić, and M. D. Lukin, Generation and manipulation of Schrödinger cat states in Rydberg atom arrays, Science 365, 570 (2019).

[9] S. de Léséleuc, V. Lienhard, P. Scholl, D. Barredo, S. Weber, N. Lang, H. P. Büchler, Th. Lahaye, and A. Browaeys, Observation of a symmetry-protected topological phase of interacting bosons with Rydberg atoms, Science 365, 775 (2019).

[10] R. G. Cortiñas, M. Favier, B. Ravon, P. Méhaignerie, Y. Machu, J. M. Raimond, C. Sayrin, and M. Brune, Laser Trapping of Circular Rydberg Atoms, Phys. Rev. Lett. 124, 123201 (2020).

[11] F. Meinert, C. Hölzl, M. A. Nebioglu, A. D’Arnese, P. Karl, M. Dressel, and M. Scheffler, Indium tin oxide films meet circular Rydberg atoms: Prospects for novel quantum simulation schemes, Phys. Rev. Res. 2, 023192 (2020).

[12] G. Pupillo, A. Micheli, M. Boninsegni, I. Lesanovsky, and P. Zoller, Strongly Correlated Gases of Rydberg-Dressed
Atoms: Quantum and Classical Dynamics, Phys. Rev. Lett. 104, 223002 (2010).

[13] T. Macrì and T. Pohl, Rydberg dressing of atoms in optical lattices, Phys. Rev. A 89, 011402 (2014).

[14] Y. Y. Jau, A. M. Hankin, T. Keating, I. H. Deutsch, and G. W. Biedermann, Entangling atomic spins with a Rydbergdressed spin-flip blockade, Nat. Phys. 12, 71 (2016).

[15] J. Zeiher, R. van Bijnen, P. Schauß, S. Hild, J.-y. Choi, T. Pohl, I. Bloch, and C. Gross, Many-body interferometry of a Rydberg-dressed spin lattice, Nat. Phys. 12, 1095 (2016).

[16] V. Borish, O. Marković, J. A. Hines, S. V. Rajagopal, and M. Schleier-Smith, Transverse-Field Ising Dynamics in a Rydberg-Dressed Atomic Gas, Phys. Rev. Lett. 124, 063601 (2020).

[17] A. W. Glaetzle, R. M. W. van Bijnen, P. Zoller, and W. Lechner, A coherent quantum annealer with Rydberg atoms, Nat. Commun. 8, 15813 (2017).

[18] I. S. Madjarov, J. P. Covey, A. L. Shaw, J. Choi, A. Kale, A. Cooper, H. Pichler, V. Schkolnik, J. R. Williams, and M. Endres, High-fidelity entanglement and detection of alkaline-earth Rydberg atoms, Nat. Phys. (16, 857 (2020).

[19] A. Signoles, T. Franz, R. Ferracini Alves, M. Gärttner, S. Whitlock, G. Zürn, and M. Weidemüller, Glassy dynamics in a disordered Heisenberg quantum spin system, arXiv:1909.11959 (2019).

[20] A. Browaeys and Th. Lahaye, Many-body physics with individually controlled Rydberg atoms, Nat. Phys. 16, 132 (2020).

[21] M. Gärttner, J. G. Bohnet, A. Safavi-Naini, M. L. Wall, J. J. Bollinger, and A. M. Rey, Measuring out-of-time-order correlations and multiple quantum spectra in a trapped-ion quantum magnet, Nat. Phys. 13, 781 (2017).

[22] K. A. Landsman, C. Figgatt, T. Schuster, N. M. Linke, B. Yoshida, N. Y. Yao, and C. Monroe, Verified quantum information scrambling, Nature 567, 61 (2019).

[23] A. Elben, B. Vermersch, R. van Bijnen, C. Kokail, T. Brydges, C. Maier, M. K. Joshi, R. Blatt, C. F. Roos, and P. Zoller, Cross-Platform Verification of Intermediate Scale Quantum Devices, Phys. Rev. Lett. 124, 010504 (2020).

[24] R. Barends et al., Digitized adiabatic quantum computing with a superconducting circuit, Nature 534, 222 (2016).

[25] C. Song, K. Xu, H. Li, Y.-R. Zhang, X. Zhang, W. Liu, Q. Guo, Z. Wang, W. Ren, J. Hao, H. Feng, H. Fan, D. Zheng, D.-W. Wang, H. Wang, and S.-Y. Zhu, Generation of multicomponent atomic Schrödinger cat states of up to 20 qubits, Science 365, 574 (2019).

[26] M. F. Parsons, A. Mazurenko, C. S. Chiu, G. Ji, D. Greif, and M. Greiner, Site-resolved measurement of the spincorrelation function in the Fermi-Hubbard model, Science 353, 1253 (2016).

[27] M. Boll, T. A. Hilker, G. Salomon, A. Omran, J. Nespolo, L. Pollet, I. Bloch, and C. Gross, Spin- and densityresolved microscopy of antiferromagnetic correlations in Fermi-Hubbard chains, Science 353, 1257 (2016).

[28] C. Gross and I. Bloch, Quantum simulations with ultracold atoms in optical lattices, Science 357, 995 (2017).

[29] M. A. Norcia, A. W. Young, W. J. Eckner, E. Oelker, J. Ye, and A. M. Kaufman, Seconds-scale coherence on an optical clock transition in a tweezer array, Science 366, 93 (2019). 
[30] I. S. Madjarov, A. Cooper, A. L. Shaw, J. P. Covey, V. Schkolnik, T. H. Yoon, J. R. Williams, and M. Endres, An Atomic-Array Optical Clock with Single-Atom Readout, Phys. Rev. X 9, 041052 (2019).

[31] R. Kaubruegger, P. Silvi, C. Kokail, R. van Bijnen, A. M. Rey, J. Ye, A. M. Kaufman, and P. Zoller, Variational Spin-Squeezing Algorithms on Programmable Quantum Sensors, Phys. Rev. Lett. 123, 260505 (2019).

[32] An alternative definition is $\mathcal{H}_{\mathrm{QND}}=H_{\text {spin }} \otimes \sigma_{c}^{z}$ with Pauli operator $\sigma_{c}^{z}$. However, we prefer the form given in Eq. (1) in light of the physical realization in Sec. II.

[33] M. A. Nielsen and I. L. Chuang, Quantum Computation and Quantum Information: 10th Anniversary Edition (Cambridge University Press, USA, 2011), 10th ed.

[34] G. Giedke, J. M. Taylor, D. D’Alessandro, M. D. Lukin, and A. Imamoğlu, Quantum measurement of a mesoscopic spin ensemble, Phys. Rev. A 74, 032316 (2006).

[35] K. M. Svore, M. B. Hastings, and M. Freedman, Faster phase estimation, Quantum Inf. Comput. 14, 306 (2014).

[36] J. S. Cotler, G. Gur-Ari, M. Hanada, J. Polchinski, P. Saad, S. H. Shenker, D. Stanford, A. Streicher, and M. Tezuka, Black holes and random matrices, J. High Energ. Phys. 2017, 118 (2017).

[37] P. Kos, M. Ljubotina, and T. Prosen, Many-Body Quantum Chaos: Analytic Connection to Random Matrix Theory, Phys. Rev. X 8, 021062 (2018).

[38] J. Šuntajs, J. Bonča, T. Prosen, and L. Vidmar, Quantum chaos challenges many-body localization, arXiv:1905. 06345 (2019).

[39] D. A. Abanin, J. H. Bardarson, G. De Tomasi, S. Gopalakrishnan, V. Khemani, S. A. Parameswaran, F. Pollmann, A. C. Potter, M. Serbyn, and R. Vasseur, Distinguishing localization from chaos: Challenges in finite-size systems, arXiv:1911.04501 (2019).

[40] C. Senko, J. Smith, P. Richerme, A. Lee, W. C. Campbell, and C. Monroe, Coherent imaging spectroscopy of a quantum many-body spin system, Science 345, 430 (2014).

[41] P. Jurcevic, P. Hauke, C. Maier, C. Hempel, B. P. Lanyon, R. Blatt, and C. F. Roos, Spectroscopy of Interacting Quasiparticles in Trapped Ions, Phys. Rev. Lett. 115, 100501 (2015).

[42] P. Roushan et al., Spectroscopic signatures of localization with interacting photons in superconducting qubits, Science 358, 1175 (2017).

[43] A. W. Glaetzle, M. Dalmonte, R. Nath, C. Gross, I. Bloch, and P. Zoller, Designing Frustrated Quantum Magnets with Laser-Dressed Rydberg Atoms, Phys. Rev. Lett. 114, 173002 (2015).

[44] R. M. W. van Bijnen and T. Pohl, Quantum Magnetism and Topological Ordering via Rydberg Dressing near Förster Resonances, Phys. Rev. Lett. 114, 243002 (2015).

[45] D. Jaksch, J. I. Cirac, P. Zoller, S. L. Rolston, R. Côté, and M. D. Lukin, Fast Quantum Gates for Neutral Atoms, Phys. Rev. Lett. 85, 2208 (2000).

[46] M. Saffman, T. G. Walker, and K. Mølmer, Quantum information with Rydberg atoms, Rev. Mod. Phys. 82, 2313 (2010).

[47] A. M. Hankin, Y.-Y. Jau, L. P. Parazzoli, C. W. Chou, D. J. Armstrong, A. J. Landahl, and G. W. Biedermann,
Two-atom Rydberg blockade using direct $6 s$ to $n p$ excitation, Phys. Rev. A 89, 033416 (2014).

[48] S. Ravets, H. Labuhn, D. Barredo, L. Béguin, Th. Lahaye, and A. Browaeys, Coherent dipole-dipole coupling between two single Rydberg atoms at an electrically-tuned Förster resonance, Nat. Phys. 10, 914 (2014).

[49] K. M. Maller, M. T. Lichtman, T. Xia, Y. Sun, M. J. Piotrowicz, A. W. Carr, L. Isenhower, and M. Saffman, Rydberg-blockade controlled-NOT gate and entanglement in a two-dimensional array of neutral-atom qubits, Phys. Rev. A 92, 022336 (2015).

[50] M. Müller, I. Lesanovsky, H. Weimer, H. P. Büchler, and P. Zoller, Mesoscopic Rydberg Gate Based on Electromagnetically Induced Transparency, Phys. Rev. Lett. 102, 170502 (2009).

[51] H. Pichler, G. Zhu, A. Seif, P. Zoller, and M. Hafezi, Measurement Protocol for the Entanglement Spectrum of Cold Atoms, Phys. Rev. X 6, 041033 (2016).

[52] G. Zhu, M. Hafezi, and T. Grover, Measurement of manybody chaos using a quantum clock, Phys. Rev. A 94, 062329 (2016).

[53] F. Grusdt, N. Y. Yao, D. Abanin, M. Fleischhauer, and E. Demler, Interferometric measurements of many-body topological invariants using mobile impurities, Nat. Commun. 7, 11994 (2016).

[54] M. Serbyn and D. A. Abanin, Loschmidt echo in manybody localized phases, Phys. Rev. B 96, 014202 (2017).

[55] Z. Xu and A. del Campo, Probing the Full Distribution of Many-Body Observables By Single-Qubit Interferometry, Phys. Rev. Lett. 122, 160602 (2019).

[56] J. T. Young, P. Bienias, R. Belyansky, A. M. Kaufman, and A. V. Gorshkov, Asymmetric blockade and multi-qubit gates via dipole-dipole interactions, arXiv:2006.02486 [quant-ph] (2020).

[57] R. Belyansky, J. T. Young, P. Bienias, Z. Eldredge, A. M. Kaufman, P. Zoller, and A. V. Gorshkov, Nondestructive Cooling of an Atomic Quantum Register via StateInsensitive Rydberg Interactions, Phys. Rev. Lett. 123, 213603 (2019).

[58] F. Haake, Quantum Signatures of Chaos, Springer Series in Synergetics (Springer, Berlin, Heidelberg, 2010), Vol. 54.

[59] A. Pal and D. A. Huse, Many-body localization phase transition, Phys. Rev. B 82, 174411 (2010).

[60] D. J. Luitz, N. Laflorencie, and F. Alet, Many-body localization edge in the random-field Heisenberg chain, Phys. Rev. B 91, 081103 (2015).

[61] L. D'Alessio, Y. Kafri, A. Polkovnikov, and M. Rigol, From quantum chaos and eigenstate thermalization to statistical mechanics and thermodynamics, Adv. Phys. 65, 239 (2016).

[62] M. Lal Mehta, Random Matrices (Academic Press, Amsterdam, 2004), 3rd ed., p.706

[63] L. Zhang, V. Khemani, and D. A. Huse, A Floquet model for the many-body localization transition, Phys. Rev. B 94, 224202 (2016).

[64] B. Bertini, P. Kos, and T. Prosen, Exact Spectral Form Factor in a Minimal Model of Many-Body Quantum Chaos, Phys. Rev. Lett. 121, 264101 (2018).

[65] A. Chan, A. De Luca, and J. T. Chalker, Solution of a Minimal Model for Many-Body Quantum Chaos, Phys. Rev. X 8, 41019 (2018). 
[66] A. Chan, A. De Luca, and J. T. Chalker, Spectral Statistics in Spatially Extended Chaotic Quantum Many-Body Systems, Phys. Rev. Lett. 121, 60601 (2018).

[67] N. Regnault and R. Nandkishore, Floquet thermalization: Symmetries and random matrix ensembles, Phys. Rev. B 93, 104203 (2016).

[68] T. Kinoshita, T. Wenger, and D. S Weiss, A quantum Newton's cradle, Nature 440, 900 (2006).

[69] M. Gring, M. Kuhnert, T. Langen, T. Kitagawa, B. Rauer, M. Schreitl, I. Mazets, D. A. Smith, E. Demler, and J. Schmiedmayer, Relaxation and prethermalization in an isolated quantum system, Science 337, 1318 (2012).

[70] T. Langen, S. Erne, R. Geiger, B. Rauer, T. Schweigler, M. Kuhnert, W. Rohringer, I. E. Mazets, T. Gasenzer, and J. Schmiedmayer, Experimental observation of a generalized Gibbs ensemble, Science 348, 207 (2015).

[71] M. Schreiber, S. S. Hodgman, P. Bordia, H. P. Luschen, M. H. Fischer, R. Vosk, E. Altman, U. Schneider, and I. Bloch, Observation of many-body localization of interacting fermions in a quasirandom optical lattice, Science 349, 842 (2015).

[72] J.-y. Choi, S. Hild, J. Zeiher, P. Schauss, A. Rubio-Abadal, T. Yefsah, V. Khemani, D. A. Huse, I. Bloch, and C. Gross, Exploring the many-body localization transition in two dimensions, Science 352, 1547 (2016).

[73] J. Smith, A. Lee, P. Richerme, B. Neyenhuis, P. W. Hess, P. Hauke, M. Heyl, D. A. Huse, and C. Monroe, Many-body localization in a quantum simulator with programmable random disorder, Nat. Phys. 12, 907 (2016).

[74] K. Xu, J.-J. Chen, Y. Zeng, Y.-R. Zhang, C. Song, W. Liu, Q. Guo, P. Zhang, D. Xu, H. Deng, K. Huang, H. Wang, X. Zhu, D. Zheng, and H. Fan, Emulating Many-Body Localization with a Superconducting Quantum Processor, Phys. Rev. Lett. 120, 050507 (2018).

[75] A. Lukin, M. Rispoli, R. Schittko, M. Eric Tai, A. M. Kaufman, S. Choi, V. Khemani, J. Léonard, and M. Greiner,
Probing entanglement in a many-body-localized system, Science 364, 256 (2019).

[76] T. Brydges, A. Elben, P. Jurcevic, B. Vermersch, C. Maier, B. P. Lanyon, P. Zoller, R. Blatt, and C. F. Roos, Probing Rényi entanglement entropy via randomized measurements, Science 364, 260 (2019).

[77] A. J. Daley, M. M. Boyd, J. Ye, and P. Zoller, Quantum Computing with Alkaline-Earth-Metal Atoms, Phys. Rev. Lett. 101, 170504 (2008).

[78] R. Mukherjee, J. Millen, R. Nath, M. P. A. Jones, and T. Pohl, Many-body physics with alkaline-earth Rydberg lattices, J. Phys. B: At. Mol. Opt. 44, 184010 (2011).

[79] D. Yang, A. Grankin, L. M. Sieberer, D. V. Vasilyev, and P. Zoller, Quantum non-demolition measurement of a manybody Hamiltonian, Nat. Commun. 11, 775 (2020).

[80] D. Sels and E. Demler, Quantum generative model for sampling many-body spectral functions, arXiv:1910.14213 (2019).

[81] D. Sels, H. Dashti, S. Mora, O. Demler, and E. Demler, Quantum approximate Bayesian computation for NMR model inference, arXiv:1910.14221 (2019).

[82] N. Šibalić, J. D. Pritchard, C. S. Adams, and K. J. Weatherill, ARC: An open-source library for calculating properties of alkali Rydberg atoms, Comput. Phys. Commun. 220, 319 (2017).

[83] B. Vermersch, A. W. Glaetzle, and P. Zoller, Magic distances in the blockade mechanism of Rydberg $p$ and $d$ states, Phys. Rev. A 91, 023411 (2015).

[84] R. van Bijnen, Ph.D. thesis, Department of Applied Physics, Technische Universiteit Eindhoven (2013).

[85] F. Reiter and A. S. Sørensen, Effective operator formalism for open quantum systems, Phys. Rev. A 85, 032111 (2012).

[86] J. Cotler, N. Hunter-Jones, J. Liu, and B. Yoshida, Chaos, complexity, and random matrices, J. High Energ. Phys. 2017, 48 (2017). 